\title{
A Study of Feynman Integrals by Micro-Differential Equations
}

\author{
Masaki Kashiwara ${ }^{1 \star}$, Takahiro Kawai ${ }^{2 \star \star}$, and Toshio Oshima ${ }^{3}$ \\ ${ }^{1}$ Department of Mathematics, Massachusetts Institute of Technology, Cambridge, MA, USA \\ 2 Department of Mathematics, Harvard University, Cambridge, MA, USA, and Research Institute \\ for Mathematical Sciences, Kyoto University, Kyoto, Japan \\ ${ }^{3}$ Department of Mathematics, College of General Education, University of Tokyo, Tokyo, Japan
}

\begin{abstract}
We use the holonomic character of Feynman integrals to describe their singularity structure explicitly in some simple cases. The results in $\S 1$ show that under moderate conditions Feynman amplitudes can be locally expressed essentially in terms of Legendre functions near the points where two positive- $\alpha$ Landau-Nakanishi surfaces meet. Related topics such as hierarchical principle in perturbation theory are also discussed in terms of holonomic systems involved. In $\S 4$ we use the concrete expressions for Feynman amplitudes obtained in $\S 1$ to discuss the validity of Sato's conjecture.
\end{abstract}

\section{Introduction}

The purpose of this paper and subsequent ones is to investigate the singularity structure of the Feynman integral by making use of the systems of microdifferential (=pseudo-differential) equations that it satisfies. In this paper we confine ourselves to the case where all the relevant particles are massive. For simplicity we also assume that they are spinless.

Since Sato [19] presented a very challenging conjecture that the $S$-matrix should satisfy a holonomic system (= maximally overdetermined system) of microdifferential equations whose characteristic variety is confined to the union of all possible Landau-Nakanishi varieties ${ }^{1}$, many results have been established to support his conjecture. See Kashiwara and Kawai [5], Kawai and Stapp [10], and references cited there. Especially, Kashiwara and Kawai [6] has established the fact that every Feynman integral satisfies a holonomic system of linear differential equations whose characteristic variety is confined to the extended Landau-

$\star \quad$ Supported in part by NSF MCS 75-2333

$\star \star \quad$ Supported in part by NSF GP 36269

1 Sato [19] implicitly assumes that there is no accumulation of the masses of relevant particles 
Nakanishi variety ${ }^{2}$. However, the utility of this fact, i.e. the holonomic character of Feynman integrals has not yet been fully demonstrated in these articles. This is mainly because the structure of the holonomic system involved is, in general, too complicated to analyze it explicitly, at least at the present stage of the theory of holonomic systems.

In view of this situation, we confine our investigation to rather simple cases in this paper and show how the holonomic character of the Feynman integral can be effectively applied to the investigation of the singularity structure of Feynman integrals in such simple cases. More concretely, our plan is as follows: In $\S 1$ we determine the singularity structure of Feynman amplitude near the points where two positive- $\alpha$ Landau-Nakanishi surfaces meet. (See Theorem 1.1 for the precise conditions.) Our results in this section and also in $\S 3$ will be of crucial importance in the global study of Feynman integrals, such as the monodromy problem. (See Speer and Westwater [22], $\S 6$. See also Regge [16,17] and Regge et al. [18] for the related topics.) In $\$ 2$ we discuss the hierarchical principle in the strict sense (Landshoff et al. [11]) in terms of the algebraic structure of the holonomic systems involved. The result in this section has a close connection with the result of Kashiwara and Kawai [7]. As a by-product of the argument in this section, one can understand the relationship between the hierarchical principle in the strict sense and that in the weak sense in terms of the algebraic structure of the holonomic systems involved. In $\S 3$ we determine the canonical form of the simple holonomic system whose characteristic variety is the conormal set of the variety which has the cuspidal singularity.

In the last section, we examine in some simple examples whether the employment of the extended Landau-Nakanishi variety is necessary or not to describe the characteristic variety of the holonomic system of micro-differential equations that a Feynman integral satisfies. The result is dependent on the spacetime dimensionality. Our argument is closely tied with the results obtained in $\S 1$.

Throughout this paper we use the same notations as in Nakanishi [13], Sato et al. [20] (hereafter referred to as S-K-K [20] for short), Kashiwara and Kawai $[6,7]$, and Kawai and Stapp [10] and do not repeat their definitions.

\section{§1. Singularity Structure of a Feynman Amplitude at Some Positive- $\alpha$ Points}

The situation discussed in this section is the following:

Let $D$ be a (oriented and connected) Feynman diagram with $n$ external lines, $n^{\prime}$ vertices and $N$ internal lines and let $F_{D}(p)$ be the Feynman integral associated with $D$, that is,

$$
\begin{aligned}
F_{D}(p) & =F_{D}\left(p_{1}, \ldots, p_{n}\right) \\
& \equiv \int \frac{\prod_{j=1}^{n^{\prime}} \delta^{v}\left(\sum_{r=1}^{n}[j: r] p_{r}+\sum_{l=1}^{N}[j: l] k_{l}\right)}{\prod_{l=1}^{N}\left(k_{l}^{2}-m_{l}^{2}+\sqrt{-10}\right)} \prod_{l=1}^{N} d^{v} k_{l} .
\end{aligned}
$$

2 This variety was introduced by Kashiwara and Kawai [6] and Kashiwara et al. [9] to describe the singularity structure of the $S$-matrix and related functions. Hereafter, we will abbreviate it to "the extended Landau variety" for short 
Here $v$ denotes the space-time dimension $[j: r]$ and $[j: l]$ are incidence numbers and $k_{l}^{2}=k_{l, 0}^{2}-\sum_{\mu=1}^{\nu-1} k_{l, \mu}^{2}$. By our assumption, $m_{l}^{2}>0$. Except in $\S 4$, we always assume $v=4$.

Though the integral is a divergent one in general, it is often the case that $F_{D}(p)$ makes sense as a microfunction, i.e. modulo regular functions (Sato [19]) and actually it is well-defined in this sense in the case discussed in this section. In fact, successive application of Corollary 2.4.2 and Theorem 2.3.1 of S-K-K [20], Chapter I easily entails that $F_{D}(p)$ is a well-defined microfunction near the uniquely reversible points defined below. Note that $F_{D}(p)$ has the form $f_{D}(p) \delta^{4}\left(\sum_{j, r}[j: r] p_{r}\right)$ if it is well-defined. The function $f_{D}(p)$ is called the Feynman amplitude.

Let $D_{1}$ be the Feynman diagram obtained by contracting exactly one internal line of $D$, say the first internal line. We may assume that $\left.[j: l]\right|_{j=1, l=1}=-1$ and $\left.[j: l]\right|_{j=2, l=1}=1$.
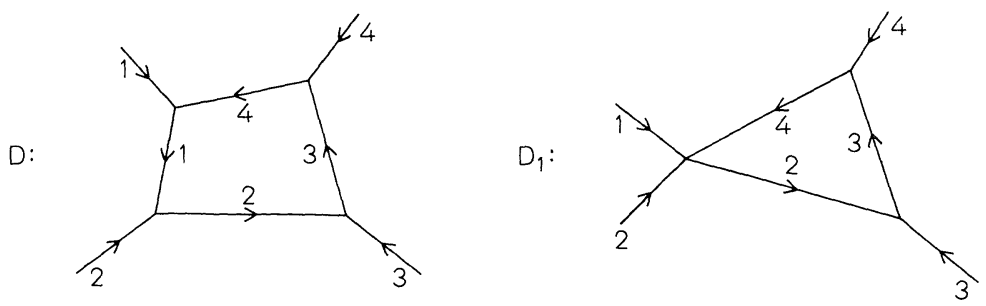

Fig. 1. Example of $D$ and $D_{1}$

Let $\mathscr{L}_{0}\left(D^{+}\right)$be the positive- $\alpha$ leading Landau-Nakanishi variety, that is, $\mathscr{L}_{0}\left(D^{+}\right)=\left\{(p ;-\sqrt{-1} u) \in \sqrt{-1} S^{*} \mathbb{R}^{4 n} ;\right.$ there exist strictly positive numbers $\alpha_{l}$ $(l=1, \ldots, N)$ and real four-vectors $k_{l}(l=1, \ldots, N)$ and $v_{j}\left(j=1, \ldots, n^{\prime}\right)$ which satisfy the following Equations (1.2a)-(1.2e)\}

$$
\begin{array}{ll}
\sum_{r=1}^{n}[j: r] p_{r}+\sum_{l=1}^{N}[j: l] k_{l}=0 & \left(j=1, \ldots, n^{\prime}\right) \\
u_{r}=\sum_{j=1}^{n^{\prime}}[j: r] v_{j} & (r=1, \ldots, n) \\
\sum_{j=1}^{n^{\prime}}[j: l] v_{j}=\alpha_{l} k_{l} & (l=1, \ldots, N) \\
k_{l}^{2}-m_{l}^{2}=0 & (l=1, \ldots, N) \\
\alpha_{l}>0 & (l=1, \ldots, N)
\end{array}
$$

In order to simplify the expression, we introduce the following notation.

Definition 1.1. We define $j(r)\left[j^{+}(l), j^{-}(l)\right.$, respectively] as the index $j$ such that $[j: r] \neq 0\left([j: l]=+1,[j: l]=-1\right.$, respectively). We also define $\varepsilon_{r}$ by $[j(r): r]$. 
If we use this notation, (1.2b) and (1.2c) are simplified as follows:

$$
\begin{array}{ll}
u_{r}=\varepsilon_{r} v_{j(r)} & (r=1, \ldots, n), \\
v_{j^{+}(l)}-v_{j^{-}(l)}=\alpha_{l} k_{l} & (l=1, \ldots, N) .
\end{array}
$$

Similarly one defines $\mathscr{L}_{0}\left(D_{1}^{+}\right)$by the following equation:

$$
\begin{array}{ll}
\sum_{j=1}^{2}\left(\sum_{r=1}^{n}[j: r] p_{r}+\sum_{l=2}^{N}[j: l] k_{l}\right)=0, \\
\sum_{r=1}^{n}[j: r] p_{r}+\sum_{l=2}^{N}[j: l] k_{l}=0 & \left(j=3, \ldots, n^{\prime}\right), \\
u_{r}=\varepsilon_{r} v_{j(r)} & (r=1, \ldots, n), \\
\sum_{j=1}^{n^{\prime}}[j: l] v_{j}=\alpha_{l} k_{l} & (l=1, \ldots, N), \\
k_{l}^{2}-m_{l}^{2}=0 & (l=2, \ldots, N), \\
\alpha_{l}>0, \quad \alpha_{1}=0 & (l=2, \ldots, N) .
\end{array}
$$

It is clear that $\mathscr{L}_{0}\left(D^{+}\right)$is a subset of positive- $\alpha$ Landau-Nakanishi variety $\mathscr{L}\left(D^{+}\right)$ associated with $D$. Recall that $\mathscr{L}\left(D^{+}\right)$is defined by

$$
\begin{array}{ll}
\sum_{r=1}^{n}[j: r] p_{r}+\sum_{l=1}^{N}[j: l] k_{l}=0 & \left(j=1, \ldots, n^{\prime}\right), \\
u_{r}=\varepsilon_{r} v_{j(r)} & (r=1, \ldots, n), \\
\sum_{j=1}^{n^{\prime}}[j: l] v_{j}=\alpha_{l} k_{l} & (l=1, \ldots, N), \\
\alpha_{l}\left(k_{l}^{2}-m_{l}^{2}\right)=0 & (l=1, \ldots, N), \\
\alpha_{l} \geqq 0 & (l=1, \ldots, N) .
\end{array}
$$

We use the symbol $\mathscr{L}(D)$ to denote the variety defined by Equations (1.4a) through $(1.4 \mathrm{~d})$. We denote by $(\mathscr{L}(D))^{\mathbb{C}}$ its complexification. A point $\left(p_{0} ;-\sqrt{-1} u_{0}\right)$ of $\mathscr{L}\left(D^{+}\right)$is called uniquely reversible after Kawai and Stapp [10], if and only if the following condition is satisfied:

Equations $^{3}(1.4 \mathrm{a})$ through $(1.4 \mathrm{~d})$ supplemented by the additional condition that $\operatorname{Re} \alpha_{l}>0(l=2, \ldots, N)$ define in a complex neighborhood of $\left(p_{0} ;-\sqrt{-1} u_{0}\right)$ a variety which has the form $\mathscr{L}_{1} \cup \mathscr{L}_{2}$ with nonsingular $\mathscr{L}_{1}$ and $\mathscr{L}_{2}$ crossing normally and whose defining ideal is reduced.

3 Here we consider the complexified equations in that every quantity is allowed to be complex 
Note that condition (UR) is satisfied for a point $\left(p_{0} ;-\sqrt{-1} u_{0}\right)$ in $\overline{\mathscr{L}_{0}\left(D^{+}\right)} \cap \mathscr{L}_{0}\left(D_{1}^{+}\right)$if the diagram $D$ is external in the sense that at least one external line is attached to each vertex of $D^{4}$.

Now recall the well-known fact that $\delta^{4}\left(\sum_{r=1}^{n}[j: r] p_{r}+\sum_{l=1}^{N}[j: l] k_{l}\right)$ and $1 /\left(k_{l}^{2}\right.$ $-m_{l}^{2}+\sqrt{-10}$ ) satisfy a holonomic system of (micro-)differential equations whose characteristic variety is confined to $\left\{(p, k ; u, w) \in P^{*}\left(\mathbb{C}^{4 n+4 N}\right)\right.$;

$$
\begin{aligned}
& \sum_{r=1}^{n}[j: r] p_{r}+\sum_{l=1}^{N}[j: l] k_{l}=0, \\
& u \equiv\left(u_{1}, \ldots, u_{r}, \ldots, u_{n}\right)=c([j: 1], \ldots,[j: r], \ldots,[j: n])^{5}
\end{aligned}
$$

and

$$
\left.w=\left(w_{1}, \ldots, w_{l}, \ldots, w_{N}\right)=c([j: 1], \ldots,[j: l], \ldots,[j: N]) \text { for } \quad c \in \mathbb{C}-\{0\}\right\}
$$

and

$$
\left\{\left(k_{l}, w_{l}\right) \in P^{*}\left(\mathbb{C}^{4}\right) ; k_{l}^{2}=m_{l}^{2}, w_{l}=\frac{c}{2} \operatorname{grad}_{k_{l}} k_{l}^{2}=c k_{l} \text { for } c \in \mathbb{C}-\{0\}\right\},
$$

respectively. Combining this fact with Proposition 4.2.4 of S-K-K [20], Chapter II, $\S 4$, one can conclude that $F_{D}(p)$ satisfies a simple holonomic system of microdifferential equations in a neighborhood of $\left(p_{0},-\sqrt{-1} u_{0}\right)$ whose characteristic variety is confined to $\mathscr{L}(D)^{\mathbb{C}}$, if $\left(p_{0} ;-\sqrt{-1} u_{0}\right)$ satisfies condition (UR $)^{6}$. Furthermore, its order on $\left(\mathscr{L}_{0}\left(D^{+}\right)\right)^{\mathbb{C}}$ and $\left(\mathscr{L}_{0}\left(D_{1}^{+}\right)\right)^{\mathbb{C}}$ are given in this case by $\alpha\left(D^{+}\right)$ $=2 n^{\prime}-\frac{3}{2} N$ and $\alpha\left(D_{1}^{+}\right)=2 n^{\prime}-\frac{3}{2} N-\frac{1}{2}$. It is also easy to see by the same reasoning that Feynman amplitude $f_{D}(p)$ satisfies a simple holonomic system of microdifferential equations in a neighborhood of $\left(p_{0},-\sqrt{-1} u_{0}\right)$ whose characteristic variety is confined to $(\mathscr{L}(D))^{\mathbb{C}}$, if $\left(p_{0} ;-\sqrt{-1} u_{0}\right)$ is uniquely reversible. Since $f_{D}(p)$ is well-defined only on $M=\left\{p=\left(p_{1}, \ldots, p_{n}\right) \in \mathbb{R}^{4 n} ; \sum_{r=1}^{n} \varepsilon_{r} p_{r}=0\right\}$, the Landau-

4 The assumption that $D_{1}$ is obtained by contracting out only one internal line of $D$ is crucial here. If several internal lines of $D$ are contracted out simultaneously, we should pay some additional attention in connection with renormalization procedure. Note also that condition (UR) is satisfied in simple contraction case if the following rank condition $(\mathrm{J})$ is satisfied.

Define $a_{j}\left(j=1, \ldots, n^{\prime}\right), b_{r}(r=1, \ldots, n), c_{l}(l=1, \ldots, N), d_{l}(l=2, \ldots, N), e_{1}$ and $f_{1}$, resp. by $\sum_{r}[j: r] p_{r}+\sum_{l}[j: l] k_{l}, u_{r}-\varepsilon_{r} v_{j(r)}, \sum_{j}[j: l] v_{j}-\alpha_{l} k_{l}, \alpha_{l}\left(k_{l}^{2}-m_{l}^{2}\right), \alpha_{1}$ and $\left(k_{1}^{2}-m_{1}^{2}\right)$, resp. Then

$$
\operatorname{rank}\left(\frac{\partial\left(a_{j}, b_{r}, c_{l}, d_{l}, e_{1}, f_{1}\right)}{\partial\left(p_{r}, u_{r}\right)}\right)=4 n^{\prime}+4 n+8 N+1
$$

holds if $\operatorname{Re} \alpha_{l}(l=2, \ldots, N)>0$

5 Here $[j: r]$ stands for the four-vector $([j: r],[j: r],[j: r],[j: r])$. This abbreviated notation is used below, if there is no fear of confusions

6 See Sato [19] for the explicit calculation for external diagram $D$ 
Nakanishi variety $\mathscr{L}(D)$ should be regarded as a subvariety of $\sqrt{-1} S^{*} M$ by introducing following convention (1.5) in considering Equation (1.4)

$$
(p ; u)=\left(p^{\prime} ; u^{\prime}\right) \quad \text { if and only if } \quad p_{r}=p_{r}^{\prime}(r=1, \ldots, n)
$$

and $u_{r}-u_{r}^{\prime}=\varepsilon_{r} c$ hold for some $c \in \mathbb{R}^{4}$.

It is clear that the order of the simple holonomic system that $f_{D}(p)$ satisfies is smaller than that for $F_{D}(p)$ by 2 , i.e. it is $2 n^{\prime}-\frac{3}{2} N-2$ on $\left(\mathscr{L}_{0}\left(D^{+}\right)\right)^{\mathbb{C}}$ and $2 n^{\prime}-\frac{3}{2} N$ $-\frac{5}{2}$ on $\left(\mathscr{L}_{0}\left(D_{1}^{+}\right)\right)^{\mathbb{C}}$.

In order to determine the singularity structure of Feynman amplitude, we first prepare the following general theorems on the structure of (solutions of) holonomic systems of micro-differential equations.

Theorem 1.1. Let $\mathfrak{M}=\mathscr{E} f \mid f=\mathscr{E}^{f} f^{7}$ be a holonomic system of micro-differential equations defined in a neighborhood of $\left\{(x ; \eta \infty) \in P^{*}\left(\mathbb{C}^{n}\right) ; x=0, \eta=(1,0, \ldots, 0)\right\}$. Assume that the characteristic variety $V$ of $\mathfrak{M}$ is the union of conormal bundles $V_{1}$ and $V_{2}$ of two non-singular hypersurfaces $H_{1}$ and $H_{2}$, respectively. Assume that $H_{1}$ and $\mathrm{H}_{2}$ are tangent mutually exactly to the second order along non-singular submanifold of codimension 2 in $\mathbb{C}^{n}$. Assume furthermore the symbol ideal $\sigma(f)$ of $\mathfrak{M}$ is reduced. Let $e_{1}$ and $e_{2}$ be the orders of the generator $f$ of $\mathfrak{M}$ on $V_{1}$ and $V_{2}$, respectively. Then, for a suitable coordinate system $x=\left(x_{1}, \ldots, x_{n}\right)$ on $\mathbb{C}^{n}, \mathfrak{M}$ is isomorphic to the following system $\mathfrak{N}_{e_{1}, e_{2}}$ by the correspondence $g=U f$ with an invertible micro-differential operator $U$ of order 0 .

$$
\mathfrak{N}_{e_{1}, e_{2}}:\left\{\begin{array}{l}
\left(x_{1} D_{x_{1}}+\frac{1}{2} x_{2} D_{x_{2}}+\frac{1}{2}\left(e_{1}+e_{2}+\frac{3}{2}\right)\right) g=0 \\
\left(\left(x_{2} D_{x_{1}}+\frac{1}{2} D_{x_{2}}\right) D_{x_{2}}+\left(e_{2}-e_{1}+\frac{1}{2}\right) D_{x_{1}}\right) g=0 \\
D_{x_{j}} g=0 \quad j=3, \ldots, n .
\end{array}\right.
$$

Proof. First we choose the local coordinate system so that $H_{1}$ and $H_{2}$ are defined by $x_{1}=0$ and $x_{1}=x_{2}^{2}$, respectively. Then by the assumptions of the theorem we see that $\sigma(f)$ is generated by $x_{1} \eta_{1}+\frac{1}{2} x_{2} \eta_{2},\left(x_{2} \eta_{1}+\frac{1}{2} \eta_{2}\right) \eta_{2}, \eta_{3}, \eta_{4}, \ldots, \eta_{n}$ because $V_{1}=\left\{(x ; \eta \infty) \in P^{*}\left(\mathbb{C}^{n}\right) ; x_{1}=\eta_{2}=\ldots=\eta_{n}=0\right\}$ and $V_{2}=\left\{(x ; \eta \infty) \in P^{*}\left(\mathbb{C}^{n}\right) ; x_{1}-x_{2}^{2}\right.$ $\left.=x_{2} \eta_{1}+\frac{1}{2} \eta_{2}=\eta_{3}=\ldots=\eta_{n}=0\right\}$. Then $\mathfrak{M}$ is of the form

$\mathfrak{M}: P_{i}(x, D) f=0, \quad i=1, \ldots, n$

7 Here $\mathscr{E}^{f}$ denotes the sheaf of micro-differential operators of finite order 
with micro-differential operators $P_{i}(x, D)$ satisfying

$$
\left\{\begin{array}{l}
\sigma\left(P_{1}\right)=x_{1} \eta_{1}+\frac{1}{2} x_{2} \eta_{2}, \\
\sigma\left(P_{2}\right)=\left(x_{2} \eta_{1}+\frac{1}{2} \eta_{2}\right) \eta_{2}, \\
\sigma\left(P_{j}\right)=\eta_{j}, \quad j=3,4, \ldots, n .
\end{array}\right.
$$

Here $\sigma(Q)$ denotes the principal symbol of $Q$ for any micro-differential operator of finite order $Q$. For simplicity we will use the notation $D_{i}$ in place of $D_{x_{i}}\left(=\partial / \partial x_{i}\right)$.

Since we can find an invertible micro-differential operator $U_{3}(x, D)$ of order 0 so that $U_{3} P_{3} U_{3}^{-1}=D_{3}$ (see Theorem 2.1.2 in Chapter II of S-K-K [20]), we may assume $P_{3}=D_{3}$ by a transformation of the generator $f$ of $\mathfrak{M}$. Moreover we can choose $P_{i}(x, D)$ so that they have the form

$$
\left\{\begin{array}{l}
P_{1}(x, D)=\left(x_{1} D_{1}+\frac{1}{2} x_{2} D_{2}\right)+\sum_{i=0}^{1} R_{1}^{i}\left(x_{2}, \ldots, x_{n}, D_{1}\right) D_{2}^{i}, \\
P_{2}(x, D)=\left(x_{2} D_{1}+\frac{1}{2} D_{2}\right) D_{2}+\sum_{i=0}^{1} R_{2}^{i}\left(x_{2}, \ldots, x_{n}, D_{1}\right) D_{2}^{i}, \\
P_{j}(x, D)=D_{j}, \quad j=3, \ldots, d, \\
P_{k}(x, D)=D_{k}+\sum_{i=0}^{1} R_{k}^{i}\left(x_{2}, \ldots, x_{n}, D_{1}\right) D_{2}^{i}, \quad k=d+1, \ldots, n,
\end{array}\right.
$$

for $d=3$ by using the same argument as in the proof of Theorem 5.1.2 in Chapter II of S-K-K [20] by the aid of the preparation theorem of Weierstrass and the theorem of Späth for micro-differential operators.

If we assume $P_{i}$ 's have the form $\left(1.8_{\mathrm{d}}\right)$ with $d=3$, then we shall find

$$
\left[D_{j}, R_{l}^{i}\right]=0, \quad i=0,1 ; \quad j=3, \ldots, d ; \quad l=1,2, d+1, \ldots, n,
$$

with $d=3$. In fact, if $\left[D_{j}, R_{l}^{0}\right]$ or $\left[D_{j}, R_{l}^{1}\right]$ were not to vanish, we should have $\sigma\left(\left[D_{j}, P_{l}\right]\right)=\sum_{i=0}^{1} r_{i}\left(x_{2}, \ldots, x_{n}, \eta_{1}\right) \eta_{2}^{i}$ with a non-zero pair $\left(r_{0}, r_{1}\right)$ of analytic functions. On the other hand, since $\left[D_{j}, P_{l}\right]$ belongs to $f$, its principal symbol vanishes on $V_{1} \cup V_{2}$. Here we find $\left(r_{0}, r_{1}\right)=0$. This is a contradiction.

Thus we see that $P_{4}$ depends only on $\left(x_{2}, x_{4}, \ldots, x_{n}, D_{1}, D_{2}, D_{4}\right)$. Therefore we can find an invertible micro-differential operator $U_{4}\left(x_{1}, x_{2}, x_{4}, \ldots, x_{n}\right.$, $\left.D_{1}, D_{2}, D_{4}, \ldots, D_{n}\right)$ of order 0 so that $U_{4} P_{4} U_{4}^{-1}=D_{4}$. Since $U_{4} D_{3} U_{4}^{-1}=D_{3}$, we may assume that $P_{j}$ equals $D_{j}$ for $j=3,4$ by a transformation of the generator of $\mathfrak{M}$ and, moreover, that $\left(1.8_{\mathrm{d}}\right)$ holds also for $d=4$.

Repeating this argument we finally find that $\left(1.8_{n}\right)$ and $\left(1.9_{n}\right)$ may be assumed. Furthermore Theorem 3.2 in Oshima [14] shows the existence of an invertible micro-differential operator $U_{1}\left(x_{1}, x_{2}, D_{1}, D_{2}\right)$ so that $U_{1} P_{1} U_{1}^{-1}=x_{1} D_{1}+\frac{1}{2} x_{2} D_{2}+\alpha$ 
with a complex number $\alpha$. Hence we may assume

$$
\left\{\begin{array}{l}
P_{1}(x, D)=x_{1} D_{1}+\frac{1}{2} x_{2} D_{2}+\alpha, \\
P_{2}(x, D)=\left(x_{2} D_{1}+\frac{1}{2} D_{2}\right) D_{2}+R_{2}^{1}\left(x_{2}, D_{1}\right) D_{2}+R_{2}^{0}\left(x_{2}, D_{1}\right), \\
P_{j}(x, D)=D_{j}, \quad j=3, \ldots, n .
\end{array}\right.
$$

Since the order of $R_{2}^{1} D_{2}+R_{2}^{0}$ is not larger than 1 , it has the form

$$
R_{2}^{1} D_{2}+R_{2}^{0}=\sum_{j=0}^{\infty} a_{j}\left(x_{2}\right) D_{1}^{-j} D_{2}+\sum_{j=0}^{\infty} b_{j}\left(x_{2}\right) D_{1}^{1-j} .
$$

Then we have

$$
\begin{aligned}
{\left[P_{1}, P_{2}\right]+P_{2}=} & {\left[x_{1} D_{1}+\frac{1}{2} x_{2} D_{2}, R_{2}^{1} D_{2}+R_{2}^{0}\right]+R_{2}^{1} D_{2}+R_{2}^{0} } \\
= & \sum_{j=0}^{\infty}\left(\frac{1}{2} x_{2} \frac{\partial a_{j}}{\partial x_{2}}+\left(j+\frac{1}{2}\right) a_{j}\right) D_{1}^{-j} \\
& +\sum_{j=0}^{\infty}\left(\frac{1}{2} x_{2} \frac{\partial b_{j}}{\partial x_{2}}+j b_{j}\right) D_{1}^{1-j}
\end{aligned}
$$

Since the operator $\left[P_{1}, P_{2}\right]+P_{2}$ belongs to $f$, we can prove that it must be zero by the same argument as that employed in the proof of (1.9).

Therefore we have

$$
\left\{\begin{array}{l}
\left(x_{2} \partial / \partial x_{2}+(2 j+1)\right) a_{j}\left(x_{2}\right)=0 \\
\left(x_{2} \partial / \partial x_{2}+2 j\right) b_{j}\left(x_{2}\right)=0
\end{array}\right.
$$

for $j=0,1,2, \ldots$. This immediately implies that $b_{0}=2 \beta$ with a complex number $\beta$ and that $a_{0}=a_{1}=a_{2}=\ldots=b_{1}=b_{2}=\ldots=0$.

Thus we have proved that $\mathfrak{M}$ is isomorphic to the system

$$
\mathfrak{N}:\left\{\begin{array}{l}
\left(x_{1} D_{1}+\frac{1}{2} x_{2} D_{2}+\alpha\right) g=0 \\
\left(\left(x_{2} D_{1}+\frac{1}{2} D_{2}\right) D_{2}+2 \beta D_{1}\right) g=0 \\
D_{j} g=0, \quad j=3, \ldots, n
\end{array}\right.
$$

by the correspondence $g=U f$ with an invertible micro-differential operator $U$ of order 0 . Since the correspondence of the generators of the system keeps their order invariant and since the order of $g$ on $V_{1}$ and that on $V_{2}$ are $\alpha-\beta-\frac{1}{2}$ and $\alpha+\beta-1$, respectively, as is shown by the direct application of the definition of the order (see Definition 4.2.3 in Chapter II of S-K-K [20]), we obtain the theorem, Q.E.D. 
The canonical form $\mathfrak{N}_{e_{1}, e_{2}}$ found in Theorem 1.1 can effectively used to find the concrete form of the solution of the system $\mathfrak{M}$, as we see in Theorem 1.2 below. In order to simplify the presentation of our results, we first prepare the following notations:

$$
\begin{aligned}
\alpha\left(e_{1}, e_{2}\right) & =\frac{1}{2}\left(e_{1}+e_{2}+\frac{3}{2}\right), \\
\beta\left(e_{1}, e_{2}\right) & =\frac{1}{2}\left(e_{2}-e_{1}+\frac{1}{2}\right), \\
G(\alpha, \beta, \gamma ; z) & \equiv \frac{\partial}{\partial \alpha} F(\alpha, \beta, \gamma ; z) .
\end{aligned}
$$

Here and in the sequel $F(\alpha, \beta, \gamma ; z)$ denotes the hypergeometric function, i.e.

$$
\begin{aligned}
& \frac{\Gamma(\gamma)}{\Gamma(\alpha) \Gamma(\beta)} \sum_{n=0}^{\infty} \frac{\Gamma(\alpha+n) \Gamma(\beta+n)}{\Gamma(\gamma+n)} \frac{z^{n}}{n !} 8 \\
& u_{1}\left(e_{1}, e_{2}\right)=\left(x_{1}+\sqrt{-10}\right)^{-\alpha\left(e_{1}, e_{2}\right)} F\left(\alpha\left(e_{1}, e_{2}\right), \beta\left(e_{1}, e_{2}\right), \frac{1}{2} ; \frac{x_{2}^{2}}{x_{1}+\sqrt{-10}}\right) \text {, } \\
& u_{2}\left(e_{1}, e_{2}\right)=x_{2}\left(x_{1}+\sqrt{-10}\right)^{-\alpha\left(e_{1}, e_{2}\right)-\frac{1}{2}} \\
& \cdot F\left(\alpha\left(e_{1}, e_{2}\right)+\frac{1}{2}, \beta\left(e_{1}, e_{2}\right)+\frac{1}{2}, \frac{3}{2} ; \frac{x_{2}^{2}}{x_{1}+\sqrt{-10}}\right), \\
& \tilde{u}_{1}\left(e_{1}, e_{2}\right) \equiv \frac{\partial u_{1}}{\partial \alpha}=-\left(x_{1}+\sqrt{-10}\right)^{-\alpha\left(e_{1}, e_{2}\right)} \log \left(x_{1}+\sqrt{-10}\right) \\
& \cdot F\left(\alpha\left(e_{1}, e_{2}\right), \beta\left(e_{1}, e_{2}\right), \frac{1}{2} ; \frac{x_{2}^{2}}{x_{1}+\sqrt{-10}}\right) \\
& +\left(x_{1}+\sqrt{-10}\right)^{-\alpha\left(e_{1}, e_{2}\right)} G\left(\alpha\left(e_{1}, e_{2}\right), \beta\left(e_{1}, e_{2}\right), \frac{1}{2} ; \frac{x_{2}^{2}}{x_{1}+\sqrt{-10}}\right) \text {, } \\
& \tilde{u}_{2}\left(e_{1}, e_{2}\right) \equiv \frac{\partial u_{2}}{\partial \alpha}=-x_{2}\left(x_{1}+\sqrt{-10}\right)^{-\alpha\left(e_{1}, e_{2}\right)-\frac{1}{2}} \\
& \cdot \log \left(x_{1}+\sqrt{-10}\right) F\left(\alpha\left(e_{1}, e_{2}\right)+\frac{1}{2}, \beta\left(e_{1}, e_{2}\right)+\frac{1}{2}, \frac{3}{2} ; \frac{x_{2}^{2}}{x_{1}+\sqrt{-10}}\right) \\
& +x_{2}\left(x_{1}+\sqrt{-10}\right)^{-\alpha\left(e_{1}, e_{2}\right)-\frac{1}{2}} \\
& \cdot G\left(\alpha\left(e_{1}, e_{2}\right)+\frac{1}{2}, \beta\left(e_{1}, e_{2}\right)+\frac{1}{2}, \frac{3}{2} ; \frac{x_{2}^{2}}{x_{1}+\sqrt{-10}}\right), \\
& v\left(e_{1}, e_{2}\right)=\frac{\Gamma\left(\alpha\left(e_{1}, e_{2}\right)\right)}{2 \Gamma\left(\beta\left(e_{1}, e_{2}\right)+\frac{1}{2}\right)} u_{1}\left(e_{1}, e_{2}\right)+\frac{\Gamma\left(\alpha\left(e_{1}, e_{2}\right)+\frac{1}{2}\right)}{\Gamma\left(\beta\left(e_{1}, e_{2}\right)\right)} u_{2}\left(e_{1}, e_{2}\right)
\end{aligned}
$$

\footnotetext{
8 We consider the analytically continued function outside $\{z \in \mathbb{C} ; z \in \mathbb{R}, z \geqq 1\}$
} 


$$
\begin{aligned}
\tilde{v}\left(e_{1}, e_{2}\right)= & \frac{\exp \left(\alpha\left(e_{1}, e_{2}\right) \pi \sqrt{-1}\right)}{2 \Gamma\left(\beta\left(e_{1}, e_{2}\right)+\frac{1}{2}\right) \Gamma\left(-\alpha\left(e_{1}, e_{2}\right)+1\right)} \tilde{u}_{1}\left(e_{1}, e_{2}\right) \\
& +\frac{\Gamma\left(\alpha\left(e_{1}, e_{2}\right)+\frac{1}{2}\right)}{\Gamma\left(\beta\left(e_{1}, e_{2}\right)\right)} u_{2}\left(e_{1}, e_{2}\right), \\
\tilde{\tilde{v}}\left(e_{1}, e_{2}\right)= & \frac{\Gamma\left(\alpha\left(e_{1}, e_{2}\right)\right)}{2 \Gamma\left(\beta\left(e_{1}, e_{2}\right)+\frac{1}{2}\right)} u_{1}\left(e_{1}, e_{2}\right)+\frac{\exp \left(\left(\alpha\left(e_{1}, e_{2}\right)+\frac{1}{2}\right) \pi \sqrt{-1}\right)}{\Gamma\left(\beta\left(e_{1}, e_{2}\right)\right) \Gamma\left(-\alpha\left(e_{1}, e_{2}\right)+\frac{1}{2}\right)} \tilde{u}_{2}\left(e_{1}, e_{2}\right) .
\end{aligned}
$$

Before stating the theorem, we list up some important properties of hyperfunctions $u_{1}\left(e_{1}, e_{2}\right)$ etc. First we examine analytic solutions of $\mathfrak{N}_{e_{1}, e_{2}}$. The equations

$$
\left\{\begin{array}{l}
\left(x_{1} D_{x_{1}}+\frac{1}{2} x_{2} D_{x_{2}}+\frac{1}{2}\left(e_{1}+e_{2}+\frac{3}{2}\right)\right) g=0 \\
D_{x_{J}} g=0, \quad j=3, \ldots, n
\end{array}\right.
$$

entail that $g$ is of the form

$$
g=x_{1}^{-\alpha} h(t)
$$

with $t=x_{2}^{2} / x_{1}$. Since

$$
\begin{aligned}
x_{2}^{2} & \left(\left(x_{2} D_{x_{1}}+\frac{1}{2} D_{x_{2}}\right) D_{x_{2}}+2 \beta D_{x_{1}}\right) x_{1}^{-\alpha} h(t) \\
= & \left.x_{1}^{-\alpha}\left(x_{2}^{2}\left(\left(x_{2} D_{x_{1}}+\frac{1}{2} D_{x_{2}}\right) D_{x_{2}}+2 \beta D_{x_{1}}\right)\right)-\alpha x_{1}^{-\alpha-1}\left(x_{2}^{3} D_{x_{2}}+2 \beta x_{2}^{2}\right)\right) h(t) \\
= & \frac{x_{1}^{-\alpha}}{2}\left(\left(x_{2} D_{x_{2}}\right)^{2}-\left(x_{2} D_{x_{2}}\right)+2\left(x_{2}^{2} D_{x_{1}}\right)\left(x_{2} D_{x_{2}}\right)\right. \\
& \left.+4 \beta x_{2}^{2} D_{x_{1}}-2 \alpha x_{1}^{-1} x_{2}^{2}\left(x_{2} D_{x_{2}}\right)-4 \alpha \beta x_{1}^{-1} x_{2}^{2}\right) h(t) \\
= & \frac{x_{1}^{-\alpha}}{2}\left(4\left(t D_{t}\right)^{2}-2\left(t D_{t}\right)+4 t\left(-t D_{t}\right)\left(t D_{t}\right)+4 \beta t\left(-t D_{t}\right)-4 \alpha t\left(t D_{t}\right)-4 \alpha \beta t\right) h(t) \\
= & 2 x_{1}^{-\alpha} t\left\{(1-t) t D_{t}^{2}+\left(\frac{1}{2}-(\alpha+\beta+1) t\right) D_{t}-\alpha \beta\right\} h(t),
\end{aligned}
$$

the function $h(t)$ is equal to

$$
C_{1} F\left(\alpha, \beta, \frac{1}{2} ; t\right)+C_{2} t^{\frac{1}{2}} F\left(\alpha+\frac{1}{2}, \beta+\frac{1}{2}, \frac{3}{2} ; t\right)
$$

with constants $C_{1}$ and $C_{2}$. Hence $u_{1}\left(e_{1}, e_{2}\right)$ and $u_{2}\left(e_{1}, e_{2}\right)$ are hyperfunction solutions of $\mathfrak{N}_{e_{1}, e_{2}}$. 
If $-\alpha \in \mathbb{N}=\{0,1,2, \ldots\}$, then $u_{1}\left(e_{1}, e_{2}\right)$ is an analytic function. Therefore the microfunction

$$
\operatorname{sp}\left(\frac{u_{1}\left(e_{1}(\alpha+t, \beta), e_{2}(\alpha+t, \beta)\right)-u_{1}\left(e_{1}(\alpha, \beta), e_{2}(\alpha, \beta)\right)}{t}\right)^{9}
$$

is a solution of $\mathfrak{N}_{e_{1}(\alpha+t, \beta), e_{2}(\alpha+t, \beta)}$. This proves that $\frac{\partial u_{1}}{\partial \alpha}\left(e_{1}, e_{2}\right) \equiv \tilde{u}_{1}\left(e_{1}, e_{2}\right)$ is a microfunction solution of $\mathfrak{N}_{e_{1}, e_{2}}$.

In the same way we can prove $\frac{\partial u_{2}}{\partial \alpha}\left(e_{1}, e_{2}\right) \equiv \tilde{u}_{2}\left(e_{1}, e_{2}\right)$ is a microfunction solution of $\mathfrak{R}_{e_{1}, e_{2}}$ when $-\alpha-\frac{1}{2} \in \mathbb{N}$.

In passing, if we set $\Lambda_{2}^{-}=\left\{(x, \sqrt{-1} \eta \infty) \in \sqrt{-1} S^{*}\left(\mathbb{R}^{n}\right) ; x_{1}=x_{2}^{2}, \eta_{2}+2 x_{2} \eta_{1}=0\right.$, $\left.\eta_{1}>0, x_{2}<0\right\}$, then in a neighborhood of $\Lambda_{2}^{-}$, we have

$$
\begin{aligned}
& \operatorname{sp}\left(u_{1}\left(e_{1}, e_{2}\right)\right)=\operatorname{sp}\left(x_{1}+\sqrt{-10}\right)^{\alpha}\left[\frac{\Gamma\left(\frac{1}{2}\right) \Gamma\left(\alpha+\beta-\frac{1}{2}\right)}{\Gamma(\alpha) \Gamma(\beta)}\left(1-\frac{x_{2}^{2}}{x_{1}+\sqrt{-1}}\right)^{\frac{1}{2}-\alpha-\beta}\right. \\
& \cdot F\left(\frac{1}{2}-\alpha, \frac{1}{2}-\beta, \frac{3}{2}-\alpha-\beta ; 1-\frac{x_{2}^{2}}{x_{1}}\right)+\frac{\Gamma\left(\frac{1}{2}\right) \Gamma\left(\frac{1}{2}-\alpha-\beta\right)}{\Gamma\left(\frac{1}{2}-\alpha\right) \Gamma\left(\frac{1}{2}-\beta\right)} \\
& \left.\cdot F\left(\alpha, \beta, \alpha+\beta+\frac{1}{2} ; 1-\frac{x_{2}^{2}}{x_{1}}\right)\right]=\frac{\Gamma\left(\frac{1}{2}\right) \Gamma\left(\alpha+\beta-\frac{1}{2}\right)}{\Gamma(\alpha) \Gamma(\beta)} x_{1}^{\beta-\frac{1}{2}} \\
& \cdot \operatorname{sp}\left\{\left(x_{1}-x_{2}^{2}+\sqrt{-10}\right)^{\frac{1}{2}-\alpha-\beta} F\left(\frac{1}{2}-\alpha, \frac{1}{2}-\beta, \frac{3}{2}-\alpha-\beta ; 1-\frac{x_{2}^{2}}{x_{1}}\right)\right\}
\end{aligned}
$$

and

$$
\begin{aligned}
\operatorname{sp} & \left(u_{2}\left(e_{1}, e_{2}\right)\right)=\operatorname{sp} x_{2}\left(x_{1}+\sqrt{-10}\right)^{-\alpha-\frac{1}{2}} \\
& \cdot\left[\frac{\Gamma\left(\frac{3}{2}\right) \Gamma\left(\alpha+\beta-\frac{1}{2}\right)}{\Gamma\left(\alpha+\frac{1}{2}\right) \Gamma\left(\beta+\frac{1}{2}\right)}\left(1-\frac{x_{2}^{2}}{x_{1}+\sqrt{-10}}\right)^{\frac{1}{2}-\alpha-\beta}\right. \\
& \cdot F\left(1-\alpha, 1-\beta, \frac{3}{2}-\alpha-\beta ; 1-\frac{x_{2}^{2}}{x_{1}}\right)+\frac{\Gamma\left(\frac{3}{2}\right) \Gamma\left(\frac{1}{2}-\alpha-\beta\right)}{\Gamma(1-\alpha) \Gamma(1-\beta)} \\
& \left.\cdot F\left(\alpha+\frac{1}{2}, \beta+\frac{1}{2}, \alpha+\beta+\frac{1}{2} ; 1-\frac{x_{2}^{2}}{x_{1}}\right)\right]=\frac{\Gamma\left(\frac{3}{2}\right) \Gamma\left(\alpha+\beta-\frac{1}{2}\right)}{\Gamma\left(\alpha+\frac{1}{2}\right) \Gamma\left(\beta+\frac{1}{2}\right)} x_{2} x_{1}^{\beta-1} \\
& \cdot \operatorname{sp}\left(\left(x_{1}-x_{2}^{2}+\sqrt{-10}\right)^{\frac{1}{2}-\alpha-\beta} F\left(1-\alpha, 1-\beta, \frac{3}{2}-\alpha-\beta ; 1-\frac{x_{2}^{2}}{x_{1}}\right)\right) \\
= & \frac{\Gamma\left(\frac{3}{2}\right) \Gamma\left(\alpha+\beta-\frac{1}{2}\right)}{\Gamma\left(\alpha+\frac{1}{2}\right) \Gamma\left(\beta+\frac{1}{2}\right)} x_{2} x_{1}^{\beta-1}\left(\frac{x_{2}^{2}}{x_{1}}\right)^{-\frac{1}{2}} \\
& \cdot \operatorname{sp}\left(\left(x_{1}-x_{2}^{2}-\sqrt{-10)^{\frac{1}{2}}-\alpha-\beta} F\left(\frac{1}{2}-\alpha, \frac{1}{2}-\beta, \frac{3}{2}-\alpha-\beta ; 1-\frac{x_{2}^{2}}{x_{1}}\right)\right) .\right.
\end{aligned}
$$

\footnotetext{
9 Here sp denotes the map from $\mathscr{B}$ to $\pi_{*} \mathscr{C}$. See S-K-K [20], Chapter I, $\S 1.5$ for details
} 
Since $x_{2} x_{1}^{\beta-1}\left(\frac{x_{2}^{2}}{x_{1}}\right)^{-\frac{1}{2}}=-x_{1}^{\beta-\frac{1}{2}}$ in a neighbourhood of $\Lambda_{2}^{-}$, we have

$\left(\mathrm{S} . \mathrm{S} . v\left(e_{1}, e_{2}\right)\right) \cap \Lambda_{2}^{-}=\emptyset$

if $-2 \alpha \notin \mathbb{N}$. The hyperfunction $\tilde{v}\left(e_{1}, e_{2}\right)\left[\tilde{\tilde{v}}\left(e_{1}, e_{2}\right)\right.$, resp. $]$ also enjoys the same property when $-\alpha \in \mathbb{N}\left(-\alpha-\frac{1}{2} \in \mathbb{N}\right.$, resp.).

Note also that $\operatorname{sp}\left(v\left(e_{1}, e_{2}\right)\right)$ can be extended to a microfunction with holomorphic parameters $e_{1}$ and $e_{2}\left(e_{1}, e_{2} \in \mathbb{C}\right)$ so that it coincides with $\operatorname{sp}\left(\tilde{v}\left(e_{1}, e_{2}\right)\right)$ $\left[\operatorname{sp}\left(\tilde{\tilde{v}}\left(e_{1}, e_{2}\right)\right)\right.$, resp. $]$, if $-\alpha \in \mathbb{N}\left(-\alpha-\frac{1}{2} \in \mathbb{N}\right.$, resp. $)$. We surmise that this property of $v\left(e_{1}, e_{2}\right)$ would be closely tied with the renormalization procedure of the Feynman amplitude: in fact, hyperfunction $v\left(e_{1}, e_{2}\right)$ cannot enjoy such a property and it means that some "infinite regular function term" appears if $-\alpha \in \mathbb{N}$ or $-\alpha-\frac{1}{2} \in \mathbb{N}$. We hope to discuss this point somewhere else.

We have so far confirmed that $\operatorname{sp}\left(u_{1}\left(e_{1}, e_{2}\right)\right)$ etc. are microfunction solutions of $\mathfrak{N}_{e_{1}, e_{2}}$. On the other hand, a suitable quantized contact transformation (S-K-K [20], Chapter II, §3.3) transforms $\mathfrak{N}_{e_{1}, e_{2}}$ into the following system $\tilde{\mathfrak{N}}_{e_{1}, e_{2}}$ near $\left(0 ; \sqrt{-1} d x_{1} \infty\right)$ :

$$
\tilde{\mathfrak{N}}_{e_{1}, e_{2}}:\left\{\begin{array}{l}
\left(x_{1} D_{x_{1}}+e_{2}+\frac{1}{2}\right) h=0 \\
\left(x_{2} D_{x_{2}}-2 \beta\left(e_{1}, e_{2}\right)+1\right) h=0 \\
D_{x_{j}} h=0, \quad j=3, \ldots, n .
\end{array}\right.
$$

Since it can be shown by the aid of the results of Kashiwara and Kawai [5] that the dimension over $\mathbb{C}$ of the microfunction solution space $\operatorname{Hom}\left(\tilde{\mathfrak{N}}_{e_{1}, e_{2}}, \mathscr{C}\right)_{\left(0 ; \sqrt{-1} d x_{1} \infty\right)}$ of $\tilde{\mathfrak{N}}_{e_{1}, e_{2}}$ considered in a neighborhood of $\left(0 ; \sqrt{-1} d x_{1} \infty\right)$ is equal to 2 , we can easily find that $\operatorname{sp}\left(u_{1}\left(e_{1}, e_{2}\right)\right)$ etc. form the basis of $\mathscr{H}_{0 m}\left(\mathfrak{N}_{e_{1}, e_{2}}, \mathscr{C}\right)_{\left(0 ; \sqrt{-1} d x_{1} \infty\right)}$. More precisely, we have the following:

If $-2 \alpha\left(e_{1}, e_{2}\right) \notin \mathbb{N}$, then $\operatorname{sp}\left(u_{1}\left(e_{1}, e_{2}\right)\right)$ and

$\operatorname{sp}\left(u_{2}\left(e_{1}, e_{2}\right)\right)$ form the basis of

$\mathscr{H}$ om $\left(\mathfrak{M}_{e_{1}, e_{2}}, \mathscr{C}\right)_{\left(0 ; \sqrt{-1} d x_{1} \infty\right)}$.

If $-\alpha\left(e_{1}, e_{2}\right) \in \mathbb{N}$, then $\operatorname{sp}\left(\tilde{u}_{1}\left(e_{1}, e_{2}\right)\right)$ and

$\operatorname{sp}\left(u_{2}\left(e_{1}, e_{2}\right)\right)$ form the basis of

$\mathscr{H} \operatorname{lom}\left(\mathfrak{N}_{e_{1}, e_{2}}, \mathscr{C}\right)_{\left(0 ; \sqrt{-1} d x_{1} \infty\right)}$.

If $-\alpha\left(e_{1}, e_{2}\right)-\frac{1}{2} \in \mathbb{N}$, then $\operatorname{sp}\left(u_{1}\left(e_{1}, e_{2}\right)\right)$ and

$\operatorname{sp}\left(\tilde{u}_{2}\left(e_{1}, e_{2}\right)\right)$ form the basis of

$\mathscr{H}$ om $\left(\mathfrak{N}_{e_{1}, e_{2}}, \mathscr{C}\right)_{\left(0 ; \sqrt{-1} d x_{1} \infty\right)}$.

By the same reasoning we also find that the dimension of the space of microfunction solutions of $\mathfrak{N}_{e_{1}, e_{2}}$ with the additional condition that their support is disjoint from $\Lambda_{2}^{-}$is 1 and that $\operatorname{sp}\left(v\left(e_{1}, e_{2}\right)\right)\left(\operatorname{sp}\left(\tilde{v}\left(e_{1}, e_{2}\right)\right)\right.$ and $\operatorname{sp}\left(\tilde{v}\left(e_{1}, e_{2}\right)\right)$, resp. $)$ is its basis if $-2 \alpha\left(e_{1}, e_{2}\right) \notin \mathbb{N}$ [if $-\alpha\left(e_{1}, e_{2}\right) \in \mathbb{N}$ and if $-\alpha\left(e_{1}, e_{2}\right)-\frac{1}{2} \in \mathbb{N}$, resp.].

Now we state our main result.

Theorem 1.2. Let $f(x)$ be a hyperfunction defined near the origin of $\mathbb{R}^{n}$. Assume that its spectrum $\mathrm{sp}(f)$ satisfies a simple holonomic system $\mathfrak{M}$ of micro-differential 
equations which satisfies the conditions imposed in Theorem 1.1. Assume that the hypersurfaces $H_{1}$ and $H_{2}$ used there are real. Assume furthermore that S.S.f is confined to the union of

$$
\Lambda_{1}=\left\{(x ; \sqrt{-1} \eta \infty) \in \sqrt{-1} S^{*}\left(\mathbb{R}^{n}\right) ; x_{1}=0, \eta_{2}=\ldots=\eta_{n}=0, \eta_{1}>0\right\}
$$

and $\Lambda_{2}^{+}=\left\{(x ; \sqrt{-1} \eta \infty) \in \sqrt{-1} S^{*}\left(\mathbb{R}^{n}\right) ; \quad x_{1}=x_{2}^{2}, \quad \eta_{2}+2 x_{2} \eta_{1}=\eta_{3}=\ldots=\eta_{n}=0\right.$, $\left.\eta_{1}>0, x_{2}>0\right\}$. Finally assume

$$
-2 \beta\left(e_{1}, e_{2}\right) \notin \mathbb{N} \text {. }
$$

Then $f(x)$ has the form described below for some analytic functions $\varphi_{j}(x)(j=0,1,2,3)$ with $\varphi_{0}(0) \neq 0$ :

Case I. $2 \alpha\left(e_{1}, e_{2}\right)-2 \notin \mathbb{N}$,

Subcase (Ia) $2 \alpha\left(e_{1}, e_{2}\right) \notin \mathbb{Z}=\{0, \pm 1, \pm 2, \ldots\}$

$$
f(x)=\varphi_{0}(x) v\left(e_{1}, e_{2}\right)+\varphi_{1}(x) v\left(e_{1}-1, e_{2}\right)+\varphi_{2}(x),
$$

Subcase (Ib) $\alpha\left(e_{1}, e_{2}\right)=\frac{1}{2}$

$$
\begin{aligned}
& f(x)=\varphi_{0}(x) v\left(e_{1}, e_{2}\right)+\varphi_{1}(x) \tilde{v}\left(e_{1}-1, e_{2}\right)+\varphi_{2}(x) \\
& \left(=\varphi_{0}(x) v\left(e_{1},-e_{1}-\frac{1}{2}\right)+\varphi_{1}(x) \tilde{v}\left(e_{1}-1,-e_{1}-\frac{1}{2}\right)+\varphi_{2}(x)\right),
\end{aligned}
$$

Subcase (Ic) $-\alpha\left(e_{1}, e_{2}\right) \in \mathbb{N}$

$$
f(x)=\varphi_{0}(x) \tilde{v}\left(e_{1}, e_{2}\right)+\varphi_{1}(x) \tilde{\tilde{v}}\left(e_{1}-1, e_{2}\right)+\varphi_{2}(x),
$$

Subcase (Id) $-\alpha\left(e_{1}, e_{2}\right)-\frac{1}{2} \in \mathbb{N}$

$$
f(x)=\varphi_{0}(x) \tilde{v}\left(e_{1}, e_{2}\right)+\varphi_{1}(x) \tilde{v}\left(e_{1}-1, e_{2}\right)+\varphi_{2}(x) .
$$

Case II. $\quad \alpha\left(e_{1}, e_{2}\right) \in \mathbb{N}^{+}=\{1,2,3, \ldots\}$

$$
\begin{aligned}
f(x)= & \varphi_{0}(x) v\left(e_{1}, e_{2}\right)+\varphi_{1}(x) v\left(e_{1}-1, e_{2}\right)+\varphi_{3}(x) \tilde{v}\left(-\beta\left(e_{1}, e_{2}\right)-\frac{1}{2}, \beta\left(e_{1}, e_{2}\right)-1\right) \\
& +\varphi_{2}(x) .
\end{aligned}
$$

Case III. $\alpha\left(e_{1}, e_{2}\right)-\frac{1}{2} \in \mathbb{N}^{+}$

$$
\begin{aligned}
f(x)= & \varphi_{0}(x) v\left(e_{1}, e_{2}\right)+\varphi_{1}(x) v\left(e_{1}-1, e_{2}\right)+\varphi_{3}(x) \tilde{\tilde{v}}\left(-\beta\left(e_{1}, e_{2}\right)-1, \beta\left(e_{1}, e_{2}\right)-\frac{1}{2}\right) \\
& +\varphi_{2}(x) .
\end{aligned}
$$

Remark. Assumption (1.25) is imposed to eliminate the trivial case where the singularity spectrum of $f(x)$ contained in $\Lambda_{1}$.

Proof of Theorem 1.2. First we prepare the following:

Lemma 1.3. Let $g$ be the generator of the system $\mathfrak{N}_{e_{1}, e_{2}}(c f .(1.6))$ and let $P\left(x, D_{x}\right)$ be an arbitrary micro-differential operator defined in a neighbourhood of $\left\{(x ; \eta \infty) \in P^{*}\left(\mathbb{C}^{n}\right) ; x=0, \eta=(1,0, \ldots, 0)\right\}$. Then $P\left(x, D_{x}\right) g$ has the following expression with analytic functions $\psi_{0}(x), \psi_{1}(x)$ and $\psi_{2}(x)$ according to the classification used in Theorem 1.2.

Case I. $\quad P\left(x, D_{x}\right) g=\psi_{0}(x) g+\psi_{1}(x) D_{x_{2}} D_{x_{1}}^{-1} g$.

Case II. $\quad P\left(x, D_{x}\right) g=\psi_{0}(x) g+\psi_{1}(x) D_{x_{2}} D_{x_{1}}^{-1} g+\psi_{2}(x) D_{x_{1}}^{-\alpha\left(e_{1}, e_{2}\right)} g$.

Case III. $P\left(x, D_{x}\right) g=\psi_{0}(x) g+\psi_{1}(x) D_{x_{2}} D_{x_{1}}^{-1} g+\psi_{2}(x) D_{x_{2}} D_{x_{2}}^{-\frac{1}{2}-\alpha\left(e_{1}, e_{2}\right)} g$.

Before giving the proof of Lemma 1.3, we show how Theorem 1.2 follows from Theorem 1.1 combined with Lemma 1.3. Since the arguments for Cases I and III are the same as that for Case II, we shall discuss Case II. (Actually, the argument for Case I is a little easier, technically speaking.) 
By virtue of Theorem 1.1, we know that $U\left(x, D_{x}\right) \operatorname{sp}(f(x))$ is a microfunction solution of $\mathfrak{N}_{e_{1}, e_{2}}$ supported by $\Lambda_{1} \cup \Lambda_{2}^{-}$. Therefore we can find a non-zero constant $C_{0}$ such that

$$
\operatorname{sp}(f(x))=U^{-1}\left(x, D_{x}\right) C_{0} \operatorname{sp}\left(v\left(e_{1}, e_{2}\right)\right)
$$

holds. On the other hand we have the expression

$$
U^{-1}\left(x, D_{x}\right) g=\psi_{0}(x) g+\psi_{1}(x) D_{x_{2}} D_{x_{1}}^{-1} g+\psi_{2}(x) D_{x_{1}}^{-\alpha\left(e_{1}, e_{2}\right)} g
$$

by Lemma 1.3. Furthermore $D_{x_{1}}^{-j} g$ (resp. $D_{x_{2}} D_{x_{1}}^{-1} g$ ) is a generator of the system $\mathfrak{N}_{e_{1}-j, e_{2}-j}$ (resp. $\left.\mathfrak{N}_{e_{1}-1, e_{2}}\right)$. Therefore we have

$$
\begin{aligned}
U^{-1}\left(x, D_{x}\right) C_{0} \operatorname{sp}\left(v\left(e_{1}, e_{2}\right)\right)= & \psi_{0}(x) C_{0} \operatorname{sp}\left(v\left(e_{1}, e_{2}\right)\right)+\psi_{1}(x) C_{1} \operatorname{sp}\left(v\left(e_{1}-1, e_{2}\right)\right) \\
& +\psi_{2}(x) C_{2} \operatorname{sp}\left(\tilde{v}\left(e_{1}-\alpha\left(e_{1}, e_{2}\right), e_{2}-\alpha\left(e_{1}, e_{2}\right)\right)\right)
\end{aligned}
$$

for some constants $C_{1}$ and $C_{2}$. This implies that the hyperfunction $f(x)-\psi_{0}(x)$ . $C_{0} v\left(e_{1}, e_{2}\right)-\psi_{1}(x) C_{1} v\left(e_{1}-1, e_{2}\right)-\psi_{2}(x) C_{2} \tilde{v}\left(-\beta\left(e_{1}, e_{2}\right)-\frac{1}{2}, \beta\left(e_{1}, e_{2}\right)-1\right)$ is analytic, because its singularity spectrum is empty. Hence $f(x)$ has the required form. Moreover we note that $\psi_{0}(0) \neq 0$ in (1.26) since $U\left(x, D_{x}\right)$ is an invertible micro-differential operator of order 0 . This completes the proof of Theorem 1.2.

Now we prove Lemma 1.3. Again we give the proof only for Case II. The proof for Cases I and III are the same. By the Späth-type division theorem for microdifferential operators (S-K-K [20], Chapter II, Theorem 2.2.1) we have

$$
P\left(x, D_{x}\right) g=\sum_{j=0}^{\infty} a_{j}^{0}\left(x_{2}, \ldots, x_{n}\right) D_{1}^{-j} g+\sum_{j=0}^{\infty} b_{j}^{0}\left(x_{2}, \ldots, x_{n}\right) D_{2} D_{1}^{-1-j} g .
$$

Here $a_{j}^{0}(x)$ and $b_{j}^{0}(x)$ are analytic functions satisfying

$$
a_{j}^{0}(x), b_{j}^{0}(x) \ll C^{j+1} j !\left(r-x_{1}-\ldots-x_{n}\right)^{-1}
$$

with positive constants $C$ and $r$. Here $A \gg B$ means that $A$ is a majorant series of $B$. Using the equations

$$
\begin{aligned}
\left(x_{1} D_{1}+\frac{1}{2} x_{2} D_{2}\right) D_{2}^{i} D_{1}^{-i-j} g & =D_{2}^{i} D_{1}^{-i-j}\left(x_{1} D_{1}+\frac{1}{2} x_{2} D_{2}+\frac{i}{2}+j\right) g \\
& =\left(\frac{i}{2}+j-\alpha\right) D_{2}^{i} D_{1}^{-i-j} g
\end{aligned}
$$

and

$$
D_{2}^{2} D_{1}^{-1-j} g=\left(-2 x_{2} D_{2} D_{1}^{-j}-4 \beta D_{1}^{-j}\right) g,
$$

we can inductively determine analytic functions $a_{j}^{k}(x)$ and $b_{j}^{k}(x)$ in Case II as follows :

$$
\begin{aligned}
& \left(\sum_{j=0}^{\infty} a_{j}^{k}(x) D_{1}^{-j}+\sum_{j=0}^{\infty} b_{j}^{k}(x) D_{2} D_{1}^{-1-j}\right) g=\left(a_{0}^{k}(x)+a_{\alpha}^{k}(x) D_{1}^{-\alpha}\right. \\
& +b_{0}^{k}(x) D_{2} D_{1}^{-1}+\sum_{j \neq 0, \alpha} a_{j}^{k}(x)(j-\alpha)^{-1}\left(x_{1} D_{1}+\frac{1}{2} x_{2} D_{2}\right) D_{1}^{-j}
\end{aligned}
$$




$$
\begin{aligned}
& \left.+\sum_{j=1}^{\infty} b_{j}^{k}(x)\left(\frac{1}{2}+j-\alpha\right)^{-1}\left(x_{1} D_{1}+\frac{1}{2} x_{2} D_{2}\right) D_{2} D_{1}^{-1-j}\right) g \\
= & \left(a_{0}^{k}(x)+a_{\alpha}^{k}(x) D_{1}^{-\alpha}+b_{0}^{k}(x) D_{2} D_{1}^{-1}+\sum_{0 \leqq j \neq \alpha-1}(j+1-\alpha)^{-1} a_{j+1}^{k}(x)\right. \\
& \cdot\left(x_{1} D_{1}^{-j}+\frac{1}{2} x_{2} D_{2} D_{1}^{-1-j}\right)+\sum_{j=0}^{\infty}\left(\frac{3}{2}+j-\alpha\right)^{-1} b_{j+1}^{k}(x) x_{1} D_{2} D_{1}^{-1-j} \\
& +\sum_{j=0}^{\infty}\left(\frac{3}{2}+j-\alpha\right)^{-1} b_{j+1}^{k}(x) \frac{1}{2} x_{2}\left(-2 x_{2}\right) D_{2} D_{1}^{-1-j} \\
& \left.+\sum_{j=1}^{\infty}\left(\frac{1}{2}+j-\alpha\right)^{-1} b_{j}^{k}(x) \frac{1}{2} x_{2}(-4 \beta) D_{1}^{-j}\right) g \\
= & \left(\sum_{j=0}^{\infty} a_{j}^{k+1}(x) D_{1}^{-j}+\sum_{j=0}^{\infty} b_{j}^{k+1}(x) D_{2} D_{1}^{-1-j}\right) g,
\end{aligned}
$$

where

$$
\left\{\begin{aligned}
a_{0}^{k+1}(x)= & a_{0}^{k}(x)+\left(1-\delta_{1, \alpha}\right)(1-\alpha)^{-1} a_{1}^{k}(x) x_{1}, \\
a_{\alpha}^{k+1}(x)= & a_{\alpha}^{k}(x)+a_{\alpha+1}^{k}(x) x_{1}-4 \beta b_{\alpha}^{k}(x) x_{2}, \\
a_{j}^{k+1}(x)= & \left(1-\delta_{j+1, \alpha}\right)(j+1-\alpha)^{-1} a_{j+1}^{k}(x) x_{1} \\
& -4 \beta(1+2 j-2 \alpha)^{-1} b_{j}^{k}(x) x_{2} \text { for } j \neq 0, \alpha \\
b_{0}^{k+1}(x)= & b_{0}^{k}(x)+\frac{1}{2}\left(1-\delta_{1, \alpha}\right)(1-\alpha)^{-1} a_{1}^{k}(x) x_{2} \\
& +2(3-2 \alpha)^{-1} b_{1}^{k}(x)\left(x_{1}-x_{2}^{2}\right), \\
& 1\left(1-\delta_{j+1, \alpha}\right)(j+1-\alpha)^{-1} a_{j+1}^{k}(x) x_{2} \\
b_{j}^{k+1}(x)= & +2(3+2 j-\alpha)^{-1} b_{j+1}^{k}(x)\left(x_{1}-x_{2}^{2}\right) \text { for } j \geqq 1 .
\end{aligned}\right.
$$

Hence there exists a positive constant $M$ such that if formal power series $c_{j}^{k}(x)$ satisfy

$$
\left\{\begin{array}{l}
(j+1) c_{j}^{k+1}(x) \gg M\left(x_{1}+x_{2}+x_{2}^{2}\right)\left(c_{j+1}^{k}(x)+c_{j}^{k}(x)\right) \\
c_{j}^{0}(x) \gg C^{j+1} j !\left(r-x_{1}-\ldots-x_{n}\right)^{-1}
\end{array}\right.
$$

for $j=1,2, \ldots$ and $k=0,1,2, \ldots$, then

$$
\left\{\begin{array}{l}
c_{j}^{k}(x) \gg a_{j}^{k}(x), b_{j}^{k}(x) \quad \text { for } j \neq 0, \alpha, \\
c_{\alpha}^{k}(x) \gg b_{\alpha}^{k}(x) .
\end{array}\right.
$$

Define $c_{j}^{k}(x)$ by

$$
C^{j+1} j !\left(x_{1}+\ldots+x_{n}\right)^{k}\left(\varepsilon-x_{1}-\ldots-x_{n}\right)^{-1-k}
$$

for a positive number $\varepsilon$. Then we have

$$
(j+1) c_{j}^{k+1}(x)=C^{j+1}(j+1) !\left(x_{1}+\ldots+x_{n}\right)^{k+1}\left(\varepsilon-x_{1}-\ldots-x_{n}\right)^{-2-k}
$$


and

$$
\begin{gathered}
M\left(x_{1}+x_{2}+x_{2}^{2}\right)\left(c_{j+1}^{k}(x)+c_{j}^{k}(x)\right)=M\left(C^{j+2}(j+1) !+C^{j+1} j !\right) \\
\cdot\left(x_{1}+x_{2}+x_{2}^{2}\right)\left(x_{1}+\ldots+x_{n}\right)^{k}\left(\varepsilon-x_{1}-\ldots-x_{n}\right)^{-1-k} .
\end{gathered}
$$

Now we fix a positive number $\varepsilon$ so that $\varepsilon<r$ and that

$$
\left(x_{1}+\ldots+x_{n}\right)\left(\varepsilon-x_{1}-\ldots-x_{n}\right)^{-1} \gg M(C+1)\left(x_{1}+x_{2}+x_{2}^{2}\right) \text {. }
$$

This implies that $c_{j}^{k}(x)$ defined by (1.32) satisfy (1.30). Here we note that $c_{j}^{k}(x)$ are analytic functions on

$$
V=\left\{\left(x_{1}, \ldots, x_{n}\right) \in \mathbb{C}^{n} ;\left|x_{1}\right|+\ldots+\left|x_{n}\right|<\varepsilon / 3\right\}
$$

and satisfy

$$
\sup _{x \in V}\left|c_{j}^{k}(x)\right| \leqq \frac{3}{2 \varepsilon} 2^{-k} C^{j+1} j ! .
$$

Hence it follows from (1.29) and (1.31) that $a_{0}^{k}(x), a_{\alpha}^{k}(x), a_{i}^{k}(x)(1 \leqq i \neq \alpha), b_{0}^{k}(x)$ and $b_{j}^{k}(x)(1 \leqq j)$ converge to analytic functions $\psi_{0}(x), \psi_{2}(x), 0, \psi_{1}(x)$ and 0 on $V$, respectively, as $k$ tends to infinity. Thus we have

$$
P\left(x, D_{x}\right) g=\psi_{0}(x) g+\psi_{1}(x) D_{x_{2}} D_{x_{1}}^{-1} g+\psi_{2}(x) D_{x_{1}}^{-\alpha\left(e_{1}, e_{2}\right)} g .
$$

This completes the proof of Lemma 1.3.

\section{\$2. Strict Form of the Hierarchical Principle and the Structure of Simple Holonomic System}

As Kashiwara and Kawai [6] has shown, the holonomic system of (micro)differential equations that a Feynman amplitude satisfies will, at least in principle, determine the analytic structure of the amplitude at the point far away from the physical region, e.g. its sheet structure. In order to exemplify this expected principle, we discuss the strict form of the hierarchical principle formulated in Landshoff et al. [11] (see also Eden [3], Landshoff et al. [12], and Branson [1]) from the view point of the algebraic structure of holonomic system involved. See Kashiwara and Kawai [7] for some related topics.

We first recall the strict form of the hierarchical principle: originally it claims that the discontinuity attained by encircling any higher-order singularity does not contain the lower-order singularity (Landshoff et al. [11], p. 446). In view of the micro-local discontinuity formula (Kawai and Stapp [10]), we reformulate this property in terms of holonomic systems as follows:

Let $\left(p_{0} ;-\sqrt{-1} u_{0}\right)$ be a point in $\overline{\mathscr{L}_{0}\left(D^{+}\right)} \cap \mathscr{L}_{0}\left(D^{\prime+}\right)$, where $D^{\prime}$ is obtained by contracting out some internal lines of $D$. Consider the holonomic system $\mathfrak{M}_{D}$ of micro-differential equations that the Feynman integral $F_{D}(p)$ satisfies near $\left(p_{0}\right.$; $\left.-\sqrt{-1} u_{0}\right)$ and that is the strongest one at $\left(p_{0} ;-\sqrt{-1} u_{0}\right)$ in the sense that $\mathfrak{M}_{D}$ is a collection of all the micro-differential equations that $F_{D}(p)$ satisfies in a neighborhood of $\left(p_{0} ;-\sqrt{-1} u_{0}\right)$. Then $\mathfrak{M}_{D}$ admits a quotient Module whose characteristic variety is contained in $\overline{\left(\mathscr{L}_{0}\left(D^{+}\right)^{\mathbb{C}}\right)}$. 
Note that this formulation needs no reference to the codimensionality of the Landau-Nakanishi variety projected to the base manifold.

The hierarchical principle formulated above can be most neatly dealt with by following.

Theorem 2.1. Let $\mathfrak{M}=\mathscr{E}^{f} / \mathcal{f}$ be a holonomic system of micro-differential equations whose characteristic variety $V$ satisfies the following conditions:

$$
\begin{aligned}
& V=V_{1} \cup V_{2}, \text { where } V_{j}(j=1,2) \quad \text { is non-singular. } \\
& V_{12} \equiv V_{1} \cap V_{2} \text { is non-singular and of codimension } 1 \text { in } \\
& V_{1} \text { and } V_{2} \text {, respectively. } \\
& V_{1} \quad \text { and } V_{2} \quad \text { cross normally along } V_{12} \text {, i.e. } \\
& T_{p^{*}} V_{12}=T_{p^{*}} V_{1} \cap T_{p^{*}} V_{2} \text { holds for any } p^{*} \text { in } V_{12} .
\end{aligned}
$$

Here $T_{p c} V$ denotes the tangent space to $V$ at $p^{*}$.

The symbol ideal $\sigma(f)$ of $f$ is reduced.

Let $e_{1}\left(e_{2}\right.$, respectively) be the order of $\mathfrak{M}$ on $V_{1}-V_{12}\left(V_{2}-V_{12}\right.$, respectively). Then following three claims are equivalent mutually.

There exists a quotient Module $\mathfrak{M}_{1}$ of $\mathfrak{M}$ whose characteristic variety is contained in $V_{1}$, that is, we have the following exact sequence

$$
\mathfrak{M} \rightarrow \mathfrak{M}_{1} \rightarrow 0,
$$

where the characteristic variety of $\mathfrak{M}_{1}$ is contained in $V_{1}$.

There exists a subModule $\mathfrak{M}_{2}$ of $\mathfrak{M}$ whose characteristic variety is contained in $V_{2}$.

$$
e_{1}-e_{2}-\frac{1}{2} \in \mathbb{N}=\{0,1,2, \ldots\}
$$

Remark. We can also re-formulate the hierarchical principle in the weak sense (Landshoff et al. [11]) in terms of holonomic structures as follows (cf. Kashiwara and Kawai [7] and Regge [16]):

There exists a subModule $\mathfrak{M}_{2}$ of $\mathfrak{M}$ whose characteristic variety is confined to $V_{2}$, that is, we have the following exact sequence

$$
0 \rightarrow \mathfrak{M}_{2} \rightarrow \mathfrak{M},
$$

where the characteristic variety of $\mathfrak{M}_{2}$ is contained in $V_{2}$.

In fact, this property of $\mathfrak{M}$ is equivalent to the property that there exists a micro-differential operator $Q\left(p, D_{p}\right)$ which transforms the generator of $\mathfrak{M}$ to that of $\mathfrak{M}_{2}$. In other words, the Feynman integral associated with the daughter diagram of $D$ can be expressed as $Q\left(p, D_{p}\right) F_{D}(p)$. This is the version of the hierarchical principle formulated in Kashiwara and Kawai [7].

Now, in view of Theorem 2.1 we see that the hierarchical principle in the strict sense formulated in terms of holonomic structure is equivalent to the hierarchical principle in the weak sense formulated above on the condition that the symbol 
ideal of $\mathfrak{M}$ is reduced. Of course, the hierarchical principle in the weak sense does not necessarily imply the strict one, if $\mathfrak{M}$ has a multiplicity (Kawai and Stapp [10] and Kashiwara and Kawai [8]).

Proof. By a quontized contact transformation, we may assume $\mathfrak{M}$ is

$$
\mathfrak{N}_{e_{1}, e_{2}}:\left\{\begin{array}{l}
\left(x_{1} D_{x_{1}}+\frac{1}{2} x_{2} D_{x_{2}}+\frac{1}{2}\left(e_{1}+e_{2}+\frac{3}{2}\right)\right) g\left(e_{1}, e_{2}\right)=0 \\
\left(\left(x_{2} D_{x_{1}}+\frac{1}{2} D_{x_{2}}\right) D_{x_{2}}+\left(e_{2}-e_{1}+\frac{1}{2}\right) D_{x_{1}}\right) g\left(e_{1}, e_{2}\right)=0 \\
D_{x_{j}} g\left(e_{1}, e_{2}\right)=0 \quad j=3, \ldots, n .
\end{array}\right.
$$

Now we set

$$
\begin{aligned}
& s=\left(x_{2} D_{x_{1}}+\frac{1}{2} D_{x_{2}}\right) D_{x_{1}}^{-\frac{1}{2}} \\
& t=D_{x_{2}} D_{x_{1}}^{-\frac{1}{2}} .
\end{aligned}
$$

Then we have the following lemma by direct calculation.

Lemma 2.2. 1) $\mathfrak{N}_{e_{1}, e_{2}}$ and $\mathfrak{N}_{e_{1}+1, e_{2}+1}$ are isomorphic under the correspondence $g\left(e_{1}+1, e_{2}+1\right)=D_{x_{1}} g\left(e_{1}, e_{2}\right)$.

2) If $e_{2}-e_{1} \neq \frac{1}{2}$, then $\mathfrak{N}_{e_{1}, e_{2}}$ and $\mathfrak{N}_{e_{1}+1, e_{2}}$ are isomorphic under the correspondences $g\left(e_{1}+1, e_{2}\right)=s D_{x_{1}}^{-\frac{1}{2}} g\left(e_{1}, e_{2}\right)$ and

$$
g\left(e_{1}, e_{2}\right)=\left(e_{1}-e_{2}+\frac{1}{2}\right)^{-1} t D_{x_{1}}^{-\frac{1}{2}} g\left(e_{1}+1, e_{2}\right) .
$$

Assume (2.7). Then it follows from Lemma 2.2 that we may assume $e_{1}-e_{2}=\frac{1}{2}$. In this case the surjective homomorphism

$$
\begin{aligned}
& \mathfrak{N}_{e_{1}, e_{1}-\frac{1}{2}} \rightarrow \underset{\psi}{ } \mathfrak{N}_{e_{1}}:\left\{\left(x_{1} D_{x_{1}}+e_{1}+\frac{1}{2}\right) g\left(e_{1}\right)=0\right. \\
& g\left(e_{1}, e_{2}\right) \mapsto g\left(e_{1}\right) \quad\left\{D_{x_{j}} g\left(e_{1}\right)=0 \quad j=2, \ldots, n\right.
\end{aligned}
$$

implies (2.5).

Conversely assume (2.5) or (2.6). Then we have an exact sequence

$$
0 \rightarrow \mathfrak{M}_{2} \rightarrow \mathfrak{M} \rightarrow \mathfrak{M}_{1} \rightarrow 0
$$

with a suitable system $\mathfrak{M}_{2}$ or $\mathfrak{M}_{1}$. Hence both condition (2.5) and condition (2.6) hold, as the multiplicity of $\mathfrak{M}$ along $V_{j}$ is one, and is equal to the sum of that of $\mathfrak{M}_{1}$ along $V_{j}$ and that of $\mathfrak{M}_{2}$ along $V_{j}(j=1,2)$. On the other hand, by making use of another suitable quontized contact transformation, we may assume $\mathfrak{M}$ is equal to

$$
\mathfrak{N}_{e_{1}, e_{2}}^{\prime}:\left\{\begin{array}{l}
\left(x_{1} D_{x_{1}}+e_{1}+\frac{1}{2}\right) g^{\prime}\left(e_{1}, e_{2}\right)=0, \\
\left(x_{2} D_{x_{2}}-e_{1}+e_{2}+\frac{1}{2}\right) g^{\prime}\left(e_{1}, e_{2}\right)=0, \\
D_{x_{j}} g^{\prime}\left(e_{1}, e_{2}\right)=0, \quad j=3, \ldots, n,
\end{array}\right.
$$


where

$$
V_{1}=\left\{(x ; \eta \infty) \in P^{*}\left(\mathbb{C}^{n}\right) ; x_{1}=\eta_{2}=\eta_{3}=\ldots=\eta_{n}=0\right\}
$$

and

$$
V_{2}=\left\{(x ; \eta \infty) \in P^{*}\left(\mathbb{C}^{n}\right) ; x_{1}=x_{2}=\eta_{3}=\ldots=\eta_{n}=0, \eta_{1} \neq 0\right\} .
$$

Let $f: \mathbb{C}^{n} \rightarrow \mathbb{C}$ be a map defined by the correspondence $\left(x_{1}, \ldots, x_{n}\right) \mapsto x_{1}$. We denote by $\varpi$ the canonical projection of $\mathbb{C}^{n} \underset{\mathbb{C}}{X} P^{*} \mathbb{C}$ to $P^{*} \mathbb{C}$. Set $\mathfrak{N}_{1}=\varpi_{*} \mathscr{H}_{0 m_{\mathscr{E}}}\left(\mathscr{E}_{\mathbb{C}^{n} \rightarrow \mathbb{C}}, \mathfrak{M}_{1}\right)$. Then $\mathfrak{N}_{1}$ is a system of micro-differential equations with one variable whose characteristic variety is the conormal bundle of the origin. Then Theorem 5.3.1 of Chapter II in S-K-K [20] claims that

$$
\mathscr{H} m_{\mathscr{E}_{\mathbb{C}}}\left(\mathfrak{M}_{1}, \mathscr{C}_{\mathbb{R}^{n}}\right) \simeq \mathscr{H} m_{\mathscr{E}_{\mathbb{C}}}\left(\mathfrak{N}_{1}, \mathscr{C}_{\mathbb{R}}\right) .
$$

Since the right hand side is clearly non-vanishing at $\left(0 ; \sqrt{-1} d x_{1} \infty\right)$, there exists a non-zero microfunction solution $g(x)$ of $\mathfrak{M}_{1}$, which is also a microfunction solution of $\mathfrak{N}_{e_{1}, e_{2}}^{\prime}$ whose support is contained in $\left\{(x ; \sqrt{-1} \eta \infty) \in \sqrt{-1} S^{*} \mathbb{R}^{n} ; x_{1}=\eta_{2}\right.$ $\left.=\eta_{3}=\ldots=\eta_{n}=0, \eta_{1}>0\right\}$. On the other hand, any microfunction solution of $\mathfrak{N}_{e_{1}, e_{2}}^{\prime}$ has the representation

$$
\left\{C_{1}\left(x_{2}+\sqrt{-1} 0\right)^{e_{1}-e_{2}-\frac{1}{2}}+C_{2}\left(x_{2}-\sqrt{-1} 0\right)^{e_{1}-e_{2}-\frac{1}{2}}\right\} D_{x_{1}}^{-e_{1}-\frac{3}{2}} \delta\left(x_{1}\right)
$$

with constants $C_{1}$ and $C_{2}$ at $\left(0 ; \sqrt{-1} d x_{1} \infty\right)$ in $\sqrt{-1} S^{*}\left(\mathbb{R}^{n}\right)$ if $e_{1}-e_{2}-\frac{1}{2} \notin \mathbb{N}$ and

$$
\left\{C_{1} x_{2}^{e_{1}-e_{2}-\frac{1}{2}}+C_{2} x_{2+}^{e_{1}-e_{2}-\frac{1}{2}}\right\} D_{x_{2}}^{-e_{1}-\frac{3}{2}} \delta\left(x_{1}\right)
$$

if $e_{1}-e_{2}-\frac{1}{2} \in \mathbb{N}$. Therefore the existence of $g(x)$ with the support property implies $e_{1}-e_{2}-\frac{1}{2} \in \mathbb{N}, \quad$ Q.E.D.

\section{§3. Structure of a Simple Holonomic System whose Characteristic Variety is the Conormal Set of a Hypersurface with Cuspidal Points}

An important result due to Chandler and Stapp [2] and Pham [15] asserts that the positive- $\alpha$ Landau-Nakanishi surface ( $=$ the projection to $p$-space of LandauNakanishi variety) enjoys a nice manifold property. Especially, it ensures that there is no cuspidal point in $\pi\left(\mathscr{L}_{0}\left(D^{+}\right)\right)$for any Feynman diagram $D$. Here $\pi$ denotes the projection from the cotangent bundle to the base manifold. However, it is also known that cuspidal points are really present in $\pi\left(\mathscr{L}_{0}(D)\right)$ if positive- $\alpha$ assumption is omitted. See Westwater [23] and references cited there. Note that $\pi\left(\mathscr{L}_{0}(D)\right)$ may contain singular points even if $\mathscr{L}_{0}(D)$ is non-singular. For example, $\mathscr{L}_{0}(D)$ is non-singular if $D$ is external, although $\pi\left(\mathscr{L}_{0}(D)\right)$ is known to contain a cuspidal point for the crossed square diagram: 
Fig. 2

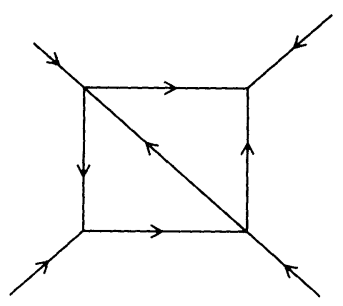

In view of the physical importance of such cuspidal points in connection with the study of Madelstam representation (Eden [4] and Landshoff et al. [12]), we show the following:

Theorem 3.1. Let $\mathfrak{M}$ be a simple holonomic system of micro-differential equations. Assume that its characteristic variety $V$ is non-singular and that its order is $e$. Assume furthermore that $\pi(V)$, the projection of $V$ to the base manifold $X$ by $\pi: P^{*} X \rightarrow X$, is of codimension 1 and that $x^{0} \in \pi(V)$ is a cuspidal point in the strict sense of Westwater [23], i.e. $\pi(V)$ can be described by the equation $x_{1}^{2}=x_{2}^{3}$ for $a$ suitable choice of local coordinate system in a neighborhood of $x^{0}$. Then $\mathfrak{M}$ is isomorphic to the following system $\mathfrak{N}_{e}$ :

$$
\mathfrak{N}_{e}:\left\{\begin{array}{l}
\left(\frac{x_{1}}{2} D_{x_{1}}+\frac{x_{2}}{3} D_{x_{2}}+e+\frac{1}{2}\right) f=0 \\
\left(\left(\frac{D_{x_{2}}}{3}\right)^{2}-x_{2}\left(\frac{D_{x_{1}}}{2}\right)^{2}\right) f=0 \\
D_{x_{J}} f=0 \quad j=3, \ldots, n=\operatorname{dim} X .
\end{array}\right.
$$

Proof. First note that the characteristic variety of the system $\mathfrak{M}$ is given by $\left\{(x ; \eta \infty) \in P^{*} X ; \frac{1}{2} x_{1} \eta_{1}+\frac{1}{3} x_{2} \eta_{2}=\left(\eta_{2} / 3\right)^{2}-x_{2}\left(\eta_{1} / 2\right)^{2}=\eta_{3}=\ldots=\eta_{n}=0\right\}$ in a neighbourhood of the point $(0 ;(1,0, \ldots, 0) \infty)$ if we use a local coordinate system $\left(x_{1}, \ldots, x_{n}\right)$ of $X$ such that $\pi(V)$ is defined by $x_{1}^{2}=x_{2}^{3}$. Then the rest of the proof of Theorem 3.1 is given in the same way as in the proof of Theorem 1.1. We leave the details to the reader.

\section{§4. Discussion on the Extended Landau Variety}

The purpose of this section is to examine whether the extended Landau variety is the smallest possible one as a characteristic variety of the holonomic system that a Feynman amplitude satisfies. Since this problem is too subtle and difficult to discuss in general, we content ourselves here with examining some simple cases where we can determine the singularity structure of the Feynman amplitude explicitly by making use of the results in $\S 1$. Our results suggest that the extended Landau variety should be the smallest possible one for generalized Feynman amplitude (Speer [21]) but that it might not be the case for the true amplitude considered in the 4-dimensional world. Note that the space-time dimension plays an important role in our discussion through the order of the holonomic system involved. Note also that the irreducible component of the extended Landau 
variety we are interested in here is Lagrangian (i.e. involutory variety with maximal codimension) and intersects with positive- $\alpha$ Landau-Nakanishi variety along a subvariety which has codimension 1 in that component. Hence the investigation of this component is crucially important from the view point of holonomic systems.

The examples we shall discuss are the Feynman amplitude $f_{D_{j}}(p)(j=1,2$, resp. $)$ associated with the diagram $D_{j}(j=1,2$, resp.) given below:
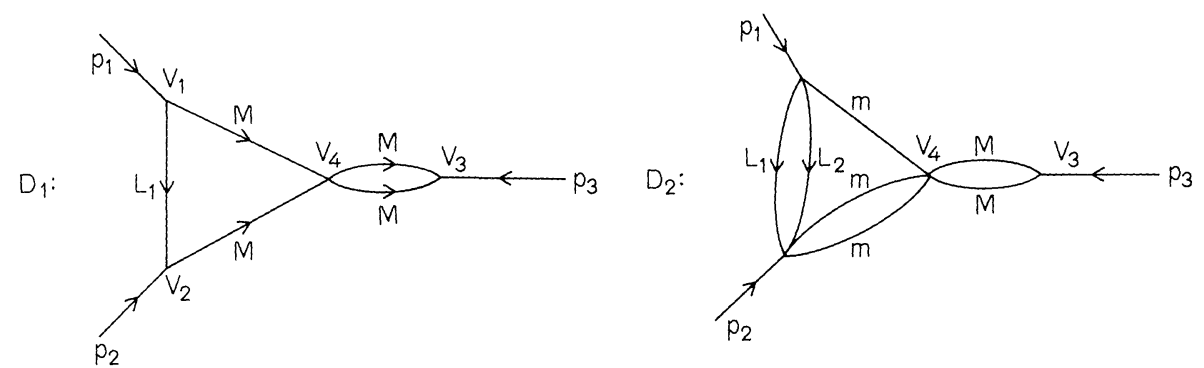

Fig. 3. $D_{1}$ : The mass associated with $L_{1}$ may be arbitrary. $D_{2}: 3 m=2 M$. The masses associated with $L_{1}$ and $L_{2}$ are arbitrary

Because of the energy-momentum conservation $\delta$-function at the vertex $V_{4}$, $f_{D_{1}}(p)$ has the following form:

$$
f_{D}\left(p_{1}, p_{2}\right) f_{D^{\prime}}\left(p_{3}\right)\left(=f_{D}\left(p_{1}, p_{2}\right) f_{D^{\prime}}\left(-\left(p_{1}+p_{2}\right)\right)\right)
$$

where $D$ and $D^{\prime}$ are specified below:

Fig. 4
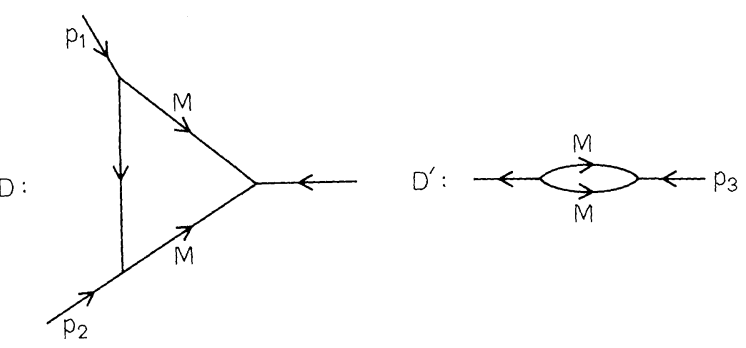

Note that $p_{1}+p_{2}=p_{3}$ holds due to the over-all conservation of energymomentum. It is also clear that $f_{D_{2}}(p)$ has the form $f_{\tilde{D}}\left(p_{1}, p_{2}\right) f_{D^{\prime}}\left(p_{3}\right)$ with $\tilde{D}$ specified below :

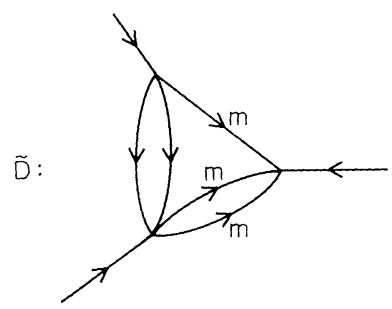

Fig. 5 
It is well-known that the positive- $\alpha$ Landau-Nakanishi variety projected to the $p$-space takes the following form described in Figure 6 below, near $\left(p_{1}+p_{2}\right)^{2}$ $=4 M^{2}$, for a suitable choice of local coordinate system $x=\left(x_{1}, \ldots, x_{n}\right)$. (See Eden et al. [4] for example.)

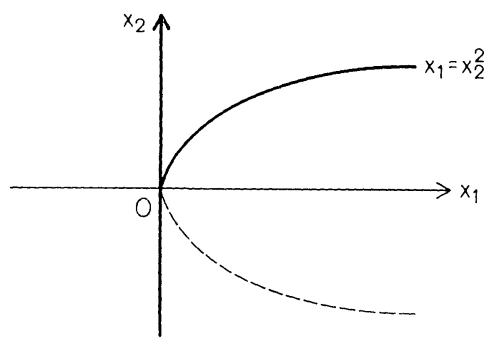

Fig. 6. The $\left(x_{3}, \ldots, x_{n}\right)$-axes are omitted

Here $n=2 v$, where $v$ stands for the space-time dimension. In Figure 6, the hypersurface $\left\{x \equiv\left(x_{1}, x_{2}, \ldots, x_{n}\right) ; x_{1}=x_{2}^{2}, x_{2}>0\right\}$ corresponds to $\pi\left(\mathscr{L}_{0}\left(D^{+}\right)\right)$and the hyperplane $\left\{x ; x_{1}=0\right\}$ corresponds to the lower order singularity of $D_{1}$, i.e., the part of positive- $\alpha$ Landau-Nakanishi variety projected to $p$-space corresponding to the contraction of $L_{1}$. The hypersurface $\left\{x ; x_{1}=0\right\}$ also corresponds to $\pi\left(\mathscr{L}_{0}\left(D^{\prime+}\right)\right)$. Note also that the extended Landau variety associated with $D_{1}$ considered near $\left(p_{1}+p_{2}\right)^{2}=4 M^{2}$ consists of the union $\left(\mathscr{L}_{0}\left(D^{+}\right)\right)^{\mathbb{C}} \cup\left(\mathscr{L}_{0}\left(D^{\prime+}\right)\right)^{\mathbb{C}} \cup \Lambda_{3}^{\mathbb{C}}$, where $\Lambda_{3}^{\mathbb{C}}$ is the complexification of $\Lambda_{3}=\{(x$; $\left.\sqrt{-1} \eta \infty) \in \sqrt{-1} S * \mathbb{R}^{n} ; x_{1}=x_{2}=0, \eta_{3}=\ldots=\eta_{n}=0\right\}$. Having this geometry in mind, we first prove Theorem 4.1, which we will use to assert that the component $\Lambda_{3}^{\mathbb{C}}$ mentioned above does not appear in the characteristic variety of the holonomic system of micro-differential equations that is satisfied by Feynman amplitude $f_{D_{1}}(p)$ considered in four-dimensional world.

Theorem 4.1. Define $\Lambda_{1}^{+}$and $\Lambda_{2}^{+}$by $\left\{(x ; \sqrt{-1} \eta \infty) \in \sqrt{-1} S^{*} \mathbb{R}^{n} ; x_{1}=0\right.$, $\left.\eta_{2}=\ldots=\eta_{n}=0, \eta_{1}>0\right\}$ and $\left\{(x ; \sqrt{-1} \eta \infty) \in \sqrt{-1} S^{*} \mathbb{R}^{n} ; x_{1}=x_{2}^{2}, \eta_{2}+2 x_{2} \eta_{1}=0\right.$, $\left.\eta_{3}=\ldots=\eta_{n}=0, \eta_{1}>0\right\}$, respectively. Denote their complexifications by $\Lambda_{1}^{\mathbb{C}}$ and $\Lambda_{2}^{\mathbb{C}}$, respectively. Let $\mathfrak{M}=\mathscr{E}^{f} / f\left(\mathfrak{M}=\mathscr{E} f / f^{\prime}\right.$, respectively) be a simple holonomic system of micro-differential equations whose characteristic variety is contained in $\Lambda_{1}^{\mathbb{C}} \cup \Lambda_{2}^{\mathbb{C}}\left(\Lambda_{1}^{\mathbb{C}}\right.$, respectively). Let $f(g$, respectively) be a hyperfunction such that $\operatorname{sp} f(\operatorname{sp} g$, respectively) satisfies $\mathfrak{M}\left(\mathfrak{M}\right.$, respectively). Assume that S.S. $f \subset \Lambda_{1}^{+} \cup \Lambda_{2}^{+}$, S.S. $f \not \subset \Lambda_{1}^{+}$ and that S.S. $g=\Lambda_{1}^{+}$. Then $\mathrm{sp}(f g)$ satisfies a holonomic system $\tilde{\mathfrak{M}}$ of micro-differential equations whose characteristic variety is confined to the union of three components $\Lambda_{1}^{\mathbb{C}}, \Lambda_{2}^{\mathbb{C}}$ and $\Lambda_{3}^{\mathbb{C}}$, where $\Lambda_{3}^{\mathbb{C}}$ is, by definition, the complexification of

$$
\Lambda_{3}=\left\{(x ; \sqrt{-1} \eta \infty) \in \sqrt{-1} S^{*} \mathbb{R}^{n} ; x_{1}=x_{2}=0, \eta_{3}=\ldots=\eta_{n}=0\right\} .
$$

Furthermore, it is contained in $\Lambda_{1}^{\mathbb{C}} \cup \Lambda_{2}^{\mathbb{C}}$, if

$$
-\left(\operatorname{ord}_{\Lambda_{1}}(\mathfrak{M})+\operatorname{ord}_{\Lambda_{1}}(\mathfrak{N})+1\right) \in \mathbb{N}
$$

holds. 
Corollary. Consider $f_{D_{1}}(p)$ in the four-dimensional world. Then $f_{D_{1}}(p)$ satisfies a holonomic system of micro-differential equations whose characteristic variety is

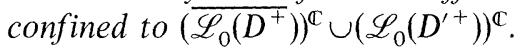

This corollary immediately follows from Theorem 4.1 with the aid of the formula given in Kawai and Stapp [10], §2.

Proof of Theorem 4.1. First we prepare the following.

Lemma 4.2. The hyperfunction $g$ in Theorem 4.1 has the form:

Case (i): $\left\{\begin{array}{l}g=\psi_{0}(x)\left(x_{1}+\sqrt{-10}\right)^{-\frac{1}{2}-e_{0}}+\psi_{1}(x) \text { if } e_{0}-\frac{1}{2} \notin \mathbb{Z}, \\ g=\psi_{0}(x)\left(x_{1}+\sqrt{-10}\right)^{-\frac{1}{2}-e_{0}} \log \left(x_{1}+\sqrt{-10}\right)+\psi_{1}(x) \\ \text { if }-e_{0}-\frac{1}{2} \in \mathbb{N},\end{array}\right.$

Case (ii): $g=\psi_{0}(x)\left(x_{1}+\sqrt{-10}\right)^{-\frac{1}{2}-e_{0}}+\psi_{1}(x)+\psi_{2}(x) \log \left(x_{1}+\sqrt{-10}\right)$

if $e_{0}-\frac{1}{2} \in \mathbb{N}$.

Here $e_{0}=\operatorname{ord}_{\Lambda_{1}} g$ and $\psi_{j}(x)(j=0,1,2)$ is an analytic function and $\psi_{0}(0) \neq 0$.

Proof. First we note following two facts a) and b):

a) There exists an invertible micro-differential operator $V\left(x, D_{x}\right)$ of order 0 such that the system $\mathfrak{N}$ is transformed into the form

$$
\mathfrak{N}_{e_{0}}:\left\{\begin{array}{l}
\left(x_{1} D_{1}+\frac{1}{2}+e_{0}\right) g^{\prime}=0 \\
D_{j} g^{\prime}=0, \quad j=2, \ldots, n
\end{array}\right.
$$

by the correspondence $g=V\left(x, D_{x}\right) g^{\prime}$ [cf. (4.2.3) of Chapter II in S-K-K [20]].

b) For the generator $g^{\prime}$ of $\mathfrak{N}_{e_{0}}$ and any micro-differential operator $Q\left(x, D_{x}\right)$ of order $0, Q\left(x, D_{x}\right) g^{\prime}$ has the form

Case (i): $Q\left(x, D_{x}\right) g^{\prime}=\psi_{0}(x) g^{\prime}$,

Case (ii): $Q\left(\dot{x}, D_{x}\right) g^{\prime}=\psi_{0}(x) g^{\prime}+\psi_{1}(x) D_{x_{1}}^{-\frac{1}{2}-e_{0}} g^{\prime}$.

In fact, by the application of Späth-type division theorem for micro-differential operators we find

$$
Q\left(x, D_{x}\right) g^{\prime}=\sum_{j=0}^{\infty} a_{j}(x) D_{x_{1}}^{-j} g^{\prime} .
$$

Then, for example, in Case (i) it suffices to set

$$
\psi_{0}(x)=\sum_{j=0}^{\infty}\left(\prod_{v=0}^{j}\left(v+\frac{1}{2}-e_{0}\right)^{-1}\right) a_{j}(x) .
$$

Hence the rest of the proof of Lemma 4.2 is the same as that of Theorem 1.2, Q.E.D. 
Lemma 4.3. Let $\mathfrak{M}(\lambda)$ be a system of differential (micro-differential, resp.) equations with a holomorphic parameter $\lambda$. Assume that the characteristic variety of $\mathfrak{M}(\lambda)$ does not depend on $\lambda$ and denote it by $V$. Let $f(x, \lambda)$ be a hyperfunction (micro-function, resp.) solution of $\mathfrak{M}(\lambda)$ with the holomorphic parameter $\lambda$. Then $(\partial f / \partial \lambda)(x, \lambda)$ satisfies a system of differential (micro-differential, resp.) equations whose characteristic variety is contained in $V$.

Proof. The assumption implies that $f(x, \lambda)-f(x, \mu)$ satisfies a system $\mathfrak{M}(\lambda, \mu)$ with the characteristic variety $V$, where $\lambda$ and $\mu$ are holomorphic parameters. Therefore $(\partial f / \partial \lambda)(x, \lambda)$ satisfies the system $\mathfrak{M}(\lambda, \lambda)$, Q.E.D.

Lemma 4.4. Set $e_{0}=\operatorname{ord}_{\Lambda_{0}}(\mathfrak{M}), e_{1}=\operatorname{ord}_{\Lambda_{1}}(\mathfrak{M})$ and $e_{2}=\operatorname{ord}_{\Lambda_{2}}(\mathfrak{M})$ and set $\alpha=\frac{1}{2}\left(e_{1}+e_{2}+\frac{3}{2}\right), \beta=\frac{1}{2}\left(e_{2}-e_{1}+\frac{1}{2}\right)$ and $\gamma=-e_{0}-\frac{1}{2}$. Let $f(x)$ be the hyperfunction solution of $\mathfrak{N}_{e_{1}, e_{2}}$ (cf. (1.6)) whose singularity spectrum is contained in $\Lambda_{1} \cup \Lambda_{2}$. Then $h(x)=\left(x_{1}+\sqrt{-10}\right)^{\gamma} f(x)$ is a hyperfunction solution of the system

$$
\mathfrak{M}(\alpha, \beta, \gamma):\left\{\begin{array}{l}
\left(x_{1} D_{x_{1}}+\frac{1}{2} x_{2} D_{x_{2}}+\alpha-\gamma\right) h=0, \\
\left(\left(x_{2} D_{x_{2}}+2(\alpha+\beta)\right)\left(x_{2} D_{x_{1}}+\frac{1}{2} D_{x_{2}}\right) D_{x_{2}}\right. \\
\left.-(\beta+\gamma) D_{x_{2}}^{2}+4 \alpha \beta D_{x_{1}}\right) h=0, \\
D_{x_{\jmath}} h=0, \quad j=3,4, \ldots, n
\end{array}\right.
$$

with the characteristic variety $\Lambda_{1}^{\mathbb{C}} \cup \Lambda_{2}^{\mathbb{C}} \cup \Lambda_{3}^{\mathbb{C}}$.

Proof. The lemma immediately follows from the following equations:

$$
\begin{aligned}
0= & \left(x_{1}+\sqrt{-1} 0\right)^{\gamma}\left(x_{1} D_{1}+\frac{1}{2} x_{2} D_{2}+\alpha\right)\left(x_{1}+\sqrt{-1} 0\right)^{-\gamma} h(x) \\
= & \left(x_{1} D_{1}+\frac{1}{2} x_{2} D_{2}+\alpha-\gamma\right) h(x) \\
0= & \left(x_{1}+\sqrt{-1} 0\right)^{\gamma} x_{1}\left(\left(x_{2} D_{1}+\frac{1}{2} D_{2}\right) D_{2}+2 \beta D_{1}\right)\left(x_{1}+\sqrt{-10}\right)^{-\gamma} h(x) \\
= & \left(x_{1}\left(x_{2} D_{1}+\frac{1}{2} D_{2}\right) D_{2}-\gamma x_{2} D_{2}+2 \beta x_{1} D_{1}-2 \beta \gamma\right) h(x) \\
= & \left(\left(x_{2} D_{1}+\frac{1}{2} D_{2}\right) D_{2} D_{1}^{-1}\left(x_{1} D_{1}+1\right)-(\gamma+1) x_{2} D_{2}+2 \beta x_{1} D_{1}-2 \beta \gamma\right) h(x) \\
= & \left(\left(x_{2} D_{1}+\frac{1}{2} D_{2}\right) D_{2} D_{1}^{-1}\left(-\frac{1}{2} x_{2} D_{2}-\alpha+\gamma+1\right)-(\gamma+1) x_{2} D_{2}\right. \\
& \left.-2 \beta\left(\frac{1}{2} x_{2} D_{2}+\alpha-\gamma\right)-2 \beta \gamma\right) h(x) \\
= & -\frac{1}{2}\left(x_{2} D_{2}\left(x_{2} D_{1}+\frac{1}{2} D_{2}\right) D_{2} D_{1}^{-1}-(\gamma-\alpha) D_{2}^{2} D_{1}^{-1}+2(\alpha+\beta) x_{2} D_{2}+4 \alpha \beta\right) h(x) .
\end{aligned}
$$


Proof of Theorem 4.1. The results in $\S 1$ and Lemma 4.2 show that $f g$ has the representation

$$
\begin{aligned}
f g= & \sum_{i=1}^{N}\left\{( a _ { 1 } ^ { i } ( x ) + ( a _ { 2 } ^ { i } ( x ) + a _ { 3 } ^ { i } ( x ) \frac { \partial } { \partial \gamma } ) ( x _ { 1 } + \sqrt { - 1 0 } ) ^ { \lambda } ) \left(b_{1}^{i}(x)\right.\right. \\
& +\left(b_{2}^{i}(x)+b_{3}^{i}(x) \frac{\partial}{\partial \alpha}\right)\left(x_{1}+\sqrt{-10}\right)^{-\alpha} F\left(\alpha, \beta, \frac{1}{2} ; \frac{x_{2}^{2}}{x_{1}+\sqrt{-1} 0}\right) \\
& +\left(b_{4}^{i}(x)+b_{5}^{i}(x) \frac{\partial}{\partial \alpha}\right)\left(x_{1}+\sqrt{-10}\right)^{-\alpha-\frac{1}{2}} \\
& \left.\cdot x_{2} F\left(\alpha+\frac{1}{2}, \beta+\frac{1}{2}, \frac{3}{2} ; \frac{x_{2}^{2}}{x_{1}+\sqrt{-1}}\right)\right\}\left.\right|_{\alpha=\alpha_{i}, \beta=\beta_{1}, \gamma=\gamma_{2}}
\end{aligned}
$$

with analytic functions $a_{j}^{i}(x)$ and $b_{j}^{i}(x)$. We note here that

$$
\left(-e_{0}-\frac{1}{2}\right)-\gamma_{i} \in \mathbb{N}
$$

and that

$$
\frac{1}{2}\left(e_{1}+e_{2}+\frac{3}{2}\right)-\alpha_{i} \in \mathbb{N} \quad \text { and } \quad \beta_{i}=\frac{1}{2}\left(e_{2}-e_{1}+\frac{1}{2}\right)
$$

or

$$
\frac{1}{2}\left(e_{1}+e_{2}+\frac{3}{2}\right)-\alpha_{i}-\frac{1}{2} \in \mathbb{N} \quad \text { and } \quad \beta_{i}=\frac{1}{2}\left(e_{2}-e_{1}+\frac{1}{2}\right)+\frac{1}{2} .
$$

Moreover,

$$
\begin{array}{lll}
a_{3}^{i}(x)=0 & \text { if } & \gamma_{i} \notin \mathbb{N}, \\
b_{3}^{i}(x)=0 & \text { if } & -\alpha_{i} \notin \mathbb{N}
\end{array}
$$

and

$$
b_{5}^{i}(x)=0 \quad \text { if } \quad-\alpha_{i}-\frac{1}{2} \notin \mathbb{N} .
$$

Hence the theorem follows from Lemmata 4.3 and 4.4 and the following lemma.

\section{Lemma 4.5. Set}

$$
h_{0}(\alpha, \beta, \gamma ; x)=\left(x_{1}+\sqrt{-1} 0\right)^{\gamma-\alpha} F\left(\alpha, \beta, \frac{1}{2} ; \frac{x_{2}^{2}}{x_{1}+\sqrt{-10}}\right)
$$

and

$$
h_{1}(\alpha, \beta, \gamma ; x)=\left(x_{1}+\sqrt{-1} 0\right)^{\gamma-\alpha-\frac{1}{2}} F\left(\alpha+\frac{1}{2}, \beta+\frac{1}{2}, \frac{3}{2} ; \frac{x_{2}^{2}}{x_{1}+\sqrt{-10}}\right) .
$$


Then the following hyperfunctions (i)-(iv) $(j=0,1)$ all satisfy holonomic systems with the characteristic variety $\Lambda_{1}^{\mathbb{C}} \cup \Lambda_{2}^{\mathbb{C}}$.

(i) $h_{j}(\alpha, \beta, \gamma ; x)$ for $\gamma-\alpha+\beta \in \mathbb{N}$,

(ii) $\partial h_{j} / \partial \gamma(\alpha, \beta, \gamma ; x)$ for $\gamma-\alpha+\beta \in \mathbb{N}, \gamma \in \mathbb{N}$,

(iii) $\partial h_{j} / \partial \alpha(\alpha, \beta, \gamma ; x)$ for $\gamma-\alpha+\beta \in \mathbb{N},-\alpha-\frac{j}{2} \in \mathbb{N}$,

(iv) $\partial^{2} h_{j} / \partial \alpha \partial \gamma(\alpha, \beta, \gamma ; x)$ for $\gamma-\alpha+\beta \in \mathbb{N},-\alpha-\frac{j}{2} \in \mathbb{N}, \gamma \in \mathbb{N}$.

Proof. Since $h_{j}(\alpha, \beta, \gamma ; x)=h_{j}(\beta, \alpha, \gamma-\alpha+\beta ; x)=x_{1}^{\gamma-\alpha+\beta} h_{j}(\beta, \alpha, 0 ; x)$ and $h_{j}(\beta, \alpha, 0 ; x)$ is a solution of $\mathfrak{N}_{-e_{1}-1, e_{2}}$ [cf. (1.6)], the hyperfunction (i) satisfies a holonomic system with the characteristic variety $\Lambda_{1}^{\mathbb{C}} \cup \Lambda_{2}^{\mathbb{C}}$.

By direct calculation we have

$$
\left(x_{1} D_{1}+\frac{1}{2} x_{2} D_{2}+\alpha-\gamma\right) \frac{\partial h_{j}}{\partial \gamma}(\alpha, \beta, \gamma ; x)=h_{j}(\alpha, \beta, \gamma ; x)
$$

and

$$
\begin{aligned}
& \left(\left(x_{2} D_{1}+\frac{1}{2} D_{2}\right) D_{2}+2 \beta D_{1}\right)\left(C_{1} h_{j}+C_{2} \frac{\partial h_{j}}{\partial \gamma}\right)(\alpha, \beta, l ; x) \\
& =\left(C_{1} l+C_{2}+C_{2} l \log \left(x_{1}+\sqrt{-10}\right)\right) x_{1}^{l-1}\left(x_{2} D_{2}+2 \beta\right) h_{j}(\alpha, \beta, 0 ; x) \\
& =\left(2 \beta+\frac{j}{2}\right)\left(\left(C_{1} l+C_{2}\right) h_{j}+C_{2} l \frac{\partial h_{j}}{\partial \gamma}\right)(\alpha, \beta+1, l-1 ; x)
\end{aligned}
$$

for $C_{1}, C_{2} \in \mathbb{C}$ and $l \in \mathbb{N}$. Hence the hyperfunction (ii) satisfies the following holonomic system with the characteristic variety $\Lambda_{1}^{\mathbb{C}} \cup \Lambda_{2}^{\mathbb{C}}$.

$$
\left\{\begin{array}{l}
\left(x_{1} D_{1}+\frac{1}{2} x_{2} D_{2}+\alpha-\gamma\right)^{2} w=0, \\
\left(\prod_{i=0}^{\gamma}\left(\left(x_{2} D_{1}+\frac{1}{2} D_{2}\right) D_{2}+2(\beta+i) D_{1}\right)\right) \\
\cdot\left(\prod_{i=0}^{\gamma-\alpha+\beta}\left(\left(x_{2} D_{1}+\frac{1}{2} D_{2}\right) D_{2}+2(\alpha+i) D_{1}\right)\right) w=0, \\
D_{k} w=0, \quad k=3, \ldots, n .
\end{array}\right.
$$

Combining the above result with Lemma 4.3 and the equations

$$
\left.\frac{\partial h_{j}(\alpha+t, \beta+t, \gamma ; x)}{\partial t}\right|_{t=0}=\frac{\partial h_{j}}{\partial \alpha}(\alpha, \beta, \gamma ; x)+\frac{\partial h_{j}}{\partial \beta}(\alpha, \beta, \gamma ; x)
$$

and

$$
\left.\frac{\partial^{2} h_{j}(\alpha+t, \beta+t, \gamma ; x)}{\partial t \partial \gamma}\right|_{t=0}=\frac{\partial^{2} h_{j}}{\partial \alpha \partial \gamma}(\alpha, \beta, \gamma ; x)+\log \left(x_{1}+\sqrt{-1} 0\right) \frac{\partial h_{j}}{\partial \beta}(\alpha, \beta, \gamma ; x),
$$

we obtain the rest of the lemma because $\partial h_{j} / \partial \beta(\alpha, \beta, \gamma ; x)$ is analytic if $-\alpha-\frac{j}{2} \in \mathbb{N}$, Q.E.D. 
In order to discuss the necessity of the conditions given in Theorem 4.1, we suppose the following conjecture of ours should be true in order to avoid some technical difficulties. We hope our results could be obtained without resorting to this conjecture.

Conjecture. Let $\mathfrak{M}=\mathscr{E}^{f} / \mathscr{J}$ be a holonomic system of micro-differential equations whose characteristic variety has the form $V_{1} \cup V_{2}\left(V_{1}\right.$, resp.), where $V_{1}$ and $V_{2}$ are non-singular and intersect normally along non-singular variety $V_{12}$. Assume that $\mathfrak{M}$ is simple both on $V_{1}-V_{12}$ and on $V_{2}-V_{12}\left(V_{1}-V_{12}\right.$, resp.). Then $\mathfrak{M}$ is isomorphic to a system $\mathscr{E}^{f} / \mathscr{J}^{\prime}$ with $\sigma\left(\mathscr{J}^{\prime}\right)$ reduced.

All the results given below depend on this conjecture at the present stage. The results depending on the conjecture stated above are indicated as $C$-Theorem etc.

C-Theorem 4.6. Assume the same conditions on $f$ and $g$ as in Theorem 4.1. Suppose the following conditions:

$$
\begin{aligned}
& \operatorname{ord}_{\Lambda_{1}}(\mathfrak{M})+\frac{1}{2} \notin \mathbb{Z} \\
& \operatorname{ord}_{\Lambda_{1}}(\mathfrak{M})+\operatorname{ord}_{\Lambda_{2}}(\mathfrak{M})+\frac{1}{2} \notin \mathbb{Z}=\{0, \pm 1, \pm 2, \ldots\}^{10} .
\end{aligned}
$$

Assume that (4.2) is not fulfilled. Then $\mathrm{sp}(f g)$ never satisfies a holonomic system of micro-differential equations whose characteristic variety is confined to $\Lambda_{1}^{\mathbb{C}} \cup \Lambda_{2}^{\mathbb{\mathbb { C }}}$.

Proof. The result in $\S 1$ and Lemma 4.2 show that the hyperfunction $f g$ has the representation

$$
\begin{aligned}
f g= & \varphi_{1}(x)\left(x_{1}+\sqrt{-10}\right)^{\gamma} w\left(e_{1}, e_{2}\right)+\varphi_{2}(x)\left(x_{1}+\sqrt{-10}\right)^{\gamma} w\left(e_{1}-1, e_{2}\right) \\
& +\varphi_{3}(x)\left(x_{1}+\sqrt{-1} 0\right)^{\gamma}+\varphi_{4}(x) w\left(e_{1}, e_{2}\right)+\varphi_{5}(x) w\left(e_{1}-1, e_{2}\right)+\varphi_{6}(x)
\end{aligned}
$$

where $\varphi_{j}(x)$ are analytic functions $(j=1, \ldots, 6)$, and $w\left(e_{1}, e_{2}\right)$ and $w\left(e_{1}-1, e_{2}\right)$ are hyperfunction solutions of $\mathfrak{N}_{e_{1}, e_{2}}$ and $\mathfrak{N}_{e_{1}-1, e_{2}}$ of (1.6), respectively, and $\gamma=-\left(\frac{1}{2}+\operatorname{ord}_{\Lambda_{1}}(\mathfrak{N})\right)$. Moreover $\varphi_{1}(0) \neq 0$ and S.S. $w\left(e_{1}, e_{2}\right)$ is not contained either in $\Lambda_{1}$ nor in $\Lambda_{2}$. In this situation we have the following lemmas:

Lemma 4.7. There exists a linear differential operator $P\left(x, D_{x}\right)$ such that

$$
\begin{aligned}
& P\left(x, D_{x}\right)\left\{\varphi_{1}(x)\left(x_{1}+\sqrt{-1} 0\right)^{\gamma} w\left(e_{1}, e_{2}\right)\right. \\
& \left.\quad+\varphi_{2}(x)\left(x_{1}+\sqrt{-10}\right)^{\gamma} w\left(e_{1}-1, e_{2}\right)\right\} \\
& =\left(x_{1}+\sqrt{-10}\right)^{\gamma} w\left(e_{1}, e_{2}\right) .
\end{aligned}
$$

10 Here ord $_{\Lambda_{1}}(\mathfrak{M})$ denotes the order of $\mathfrak{M}$ on the generic point of $\Lambda_{1}$. Conditions (4.4) and (4.5) are imposed here to avoid technical difficulties. See Remark 4.10 below 
C-Lemma 4.8. The system of micro-differential equations $\mathfrak{M}\left(\alpha\left(e_{1}, e_{2}\right), \beta\left(e_{1}, e_{2}\right)\right.$, $\left.\gamma\left(e_{1}, e_{2}\right)\right)$ in Lemma 4.4 has no quotient module whose characteristic variety is equal to $\Lambda_{1}^{\mathbb{C}} \cup \Lambda_{2}^{\mathbb{C}}$ if $\gamma-\alpha+\beta \notin \mathbb{N}$ and $\gamma \notin \mathbb{N}$.

First we show how Theorem 4.6 follows from these two lemmas.

Suppose sp $(f g)$ satisfies a system of micro-differential equations whose characteristic variety is equal to $\Lambda_{1}^{\mathbb{C}} \cup \Lambda_{2}^{\mathbb{C}}$. Since $\operatorname{sp}\left(\left(x_{1}+\sqrt{-10}\right)^{\gamma}\right), \operatorname{sp}\left(w\left(e_{1}, e_{2}\right)\right)$ and $\operatorname{sp}\left(w\left(e_{1}-1, e_{2}\right)\right)$ satisfy systems of micro-differential equations whose characteristic varieties are contained in $\Lambda_{1}^{\mathbb{C}} \cup \Lambda_{2}^{\mathbb{C}}$, so does

$$
\operatorname{sp}\left(\varphi_{1}(x)\left(x_{1}+\sqrt{-10}\right)^{\gamma} w\left(e_{1}, e_{2}\right)+\varphi_{2}(x)\left(x_{1}+\sqrt{-1} 0\right)^{\gamma} w\left(e_{1}-1, e_{2}\right)\right) .
$$

Therefore Lemma 4.7 proves that $\operatorname{sp}\left(\left(x_{1}+\sqrt{-10}\right)^{\gamma} w\left(e_{1}, e_{2}\right)\right)$ also satisfies a system of micro-differential equations whose characteristic variety is contained in $\Lambda_{1}^{\mathbb{C}} \cup \Lambda_{2}^{\mathbb{C}}$. Then the system is a quotient module of the system $\mathfrak{M}(\alpha, \beta, \gamma)$ and the assumption that S.S. $w\left(e_{1}, e_{2}\right)$ is not contains either in $\Lambda_{1}$ nor in $\Lambda_{2}$ entails that its characteristic variety should coincide with $\Lambda_{1}^{\mathbb{C}} \cup \Lambda_{2}^{\mathbb{C}}$. This contradicts to Lemma 4.8.

In order to prove Lemma 4.7 we prepare the following.

Lemma 4.9. Let $\mathcal{O}$ be the ring of the germs of analytic functions at the origin of $\mathbb{C}^{n}$. Set $X=2 x_{1} D_{x_{1}}+x_{2} D_{x_{2}}$. Let $\mathscr{J}$ be an ideal of $\mathcal{O}$ satisfying $X \mathscr{J} \subset \mathscr{J}$ and let $\phi$ be an element of $\mathscr{J}$. Then, if $\phi$ has the form $\sum_{j=0}^{\infty} \phi_{j}$ with $X \phi_{j}=j \phi_{j}, \phi_{j}$ is contained in $\mathscr{J}$ for any $j$.

Proof. Assume that $\phi_{j}=0$ for $j<k$ and that $\phi_{k} \neq 0$. Set

$$
\phi^{(l)}=\prod_{v=1}^{l}\left(1+\frac{k}{v}-\frac{X}{v}\right) \phi=\phi_{k}+(-1)^{l} \sum_{j=k+l+1}^{\infty} \frac{(j-k-1) !}{(j-k-l-1) ! l !} \phi_{j} .
$$

Then $\phi^{(l)}$ uniformly converges to $\phi_{k}$ in a neighbourhood of the origin as $l$ tends to infinity, because $(j-k-1) ! /\left((j-k-l-1) ! l ! \leqq 2^{j}\right.$. Since any ideal of $\mathcal{O}$ is closed, $\mathscr{J}$ containes $\phi_{k}$. By the same argument for $\phi-\phi_{k}$, we have $\phi_{k+1} \in \mathscr{J}$. Hence we can inductively conclude that $\phi_{j}$ is contained in $\mathscr{J}$ for $j=0,1,2, \ldots$.

Proof of Lemma 4.7. Since $\varphi_{1}(0) \neq 0$, we may assume $\varphi_{1}(x) \equiv 1$. In this case we have

$$
\begin{aligned}
& (X+2 \alpha-2 \gamma)\left(\left(x_{1}+\sqrt{-10}\right)^{\gamma} w\left(e_{1}, e_{2}\right)+\varphi_{2}(x)\left(x_{1}+\sqrt{-10}\right)^{\gamma} w\left(e_{1}-1, e_{2}\right)\right) \\
& =\left((X+1) \varphi_{2}(x)\right)\left(x_{1}+\sqrt{-1} 0\right)^{\gamma} w\left(e_{1}-1, e_{2}\right)
\end{aligned}
$$

and

$$
\begin{aligned}
& \left(X^{j} \varphi_{2}(x)\right)\left(x_{1}+\sqrt{-10}\right)^{\gamma} w\left(e_{1}-1, e_{2}\right) \\
& =(X+2 \alpha-2 \gamma-1)^{j}\left(\varphi_{2}(x)\left(x_{1}+\sqrt{-10}\right)^{\gamma} w\left(e_{1}-1, e_{2}\right)\right)
\end{aligned}
$$

for $X=2 x_{1} D_{x_{1}}+x_{2} D_{x_{2}}$. Set

$$
\mathscr{J}=\bigcup_{l=0}^{\infty}\left(\sum_{j=0}^{l} \mathcal{O} X^{j}(X+1) \varphi_{2}(x)\right) \text {. }
$$


It is clear that $\mathscr{J}$ is an ideal of $\mathcal{O}$ and that $X \mathscr{J} \subset \mathscr{J}$. Set $\varphi_{2}(x)=\sum_{j=0}^{\infty} \varphi^{(j)}$ with $X \varphi^{(j)}$ $=j \varphi^{(j)}$. Since $(X+1) \varphi_{2}(x)=\sum_{j=0}^{\infty}(j+1) \varphi^{(j)} \in \mathscr{J}$, Lemma 4.9 proves that $\varphi^{(j)} \in \mathscr{J}$ for any $j$. Therefore $\varphi_{2}(x) \in \mathscr{J}$, because $\mathscr{J}$ is closed and because $\sum_{j=0}^{l} \varphi^{(j)}$ converges $\varphi_{2}(x)$ as $l$ tends to infinity. Thus we have proved the existence of a linear differential operator

$$
Q\left(x, D_{x}\right)=\sum_{j=0}^{m} a_{j}(x) X^{j}(X+1)
$$
such that $Q\left(x, D_{x}\right) \varphi_{2}(x)=\varphi_{2}(x)$. Thus the differential operator $1-\sum_{j=0}^{m} a_{j}(x)(X+2 \alpha$
$-2 \gamma-1)^{j}(X+2 \alpha-2 \gamma)$ is a required one, Q.E.D.

Proof of Lemma 4.8. Assume $\mathfrak{M}(\alpha, \beta, \gamma)$ has a quotient module $\mathfrak{N}$ whose characteristic variety $\Lambda_{1}^{\mathbb{C}} \cup \Lambda_{2}^{\mathbb{C}}$. Since the multiplicity of $\mathfrak{M}(\alpha, \beta, \gamma)$ along $\Lambda_{1}^{\mathbb{C}}$, that along $\Lambda_{2}^{\mathbb{C}}$ and that along $\Lambda_{3}^{\mathbb{C}}$ are 2,1 and 1 , respectively, either one of the following two cases happens:

Case 1 . The multiplicity of $\mathfrak{N}$ along $\Lambda_{1}^{\mathbb{C}}$ and that along $\Lambda_{2}^{\mathbb{C}}$ are 1 .

Case 2. The multiplicity of $\mathfrak{N}$ along $\Lambda_{1}^{\mathbb{\mathbb { T }}}$ is 2 and that along $\Lambda_{2}^{\mathbb{C}}$ is 1 .

First consider Case 1 . Then the conjecture and Theorem 1.1 show that $\mathfrak{N}$ is isomorphic to $\mathfrak{N}_{e_{1}^{\prime}, e_{2}^{\prime}}$ of (2.8) with constant numbers $e_{1}^{\prime}$ and $e_{2}^{\prime}$. We use the same notation as in the proof of Theorem 2.1 in the sequel. The assumption implies the existence of a micro-differential operator $P\left(x, D_{x}\right)$ such that the correspondence $g\left(e_{1}^{\prime}, e_{2}^{\prime}\right)=P\left(x, D_{x}\right) h$ defines a surjective homomorphism of $\mathfrak{M}(\alpha, \beta, \gamma)$ onto $\mathfrak{N}_{e_{1}^{\prime}, e_{2}^{\prime}}$. Set $Q\left(x, D_{x}\right)=D_{x_{1}}^{\gamma-\alpha} P\left(x, D_{x}\right) D_{x_{1}}^{\frac{1}{2}\left(e_{1}^{\prime}+e_{2}^{\prime}+\frac{3}{2}\right)}$ and $v=\frac{1}{2}\left(e_{2}^{\prime}-e_{1}^{\prime}+\frac{1}{2}\right)$. Then the correspondence $h(\alpha, \beta, \gamma)=Q\left(x, D_{x}\right) w(v)$ defines a surjective homomorphism of the system

$$
\mathfrak{N}(\alpha, \beta, \gamma):\left\{\begin{array}{l}
\left(x_{1} D_{x_{1}}+\frac{1}{2} x_{2} D_{x_{2}}\right) h(\alpha, \beta, \gamma)=0 \\
\left(\left(s-\frac{1}{2} t\right) t s t-(\beta+\gamma) t^{2}+2(\alpha+\beta) s t+4 \alpha \beta\right) h(\alpha, \beta, \gamma)=0 \\
D_{x_{J}} h(\alpha, \beta, \gamma)=0, \quad j=3, \ldots, n
\end{array}\right.
$$

to the system

$$
\mathfrak{R}_{-v-\frac{1}{2}, v-1}:\left\{\begin{array}{l}
\left(x_{1} D_{x_{1}}+\frac{1}{2} x_{2} D_{x_{2}}\right) w(v)=0 \\
(s t+2 v) w(v)=0 \\
D_{x_{j}} w(v)=0, \quad j=3, \ldots, n .
\end{array}\right.
$$

Here $s$ and $t$ are operators defined by (2.9).

We may assume $Q$ is of the form

$$
Q\left(x, D_{x}\right)=\sum_{i, k} Q_{i, k}\left(x^{\prime}\right) s^{i} D_{x_{1}}^{\lambda+k}+\sum_{j, k} Q_{j, k}^{\prime}\left(x^{\prime}\right) t^{j} D_{x_{1}}^{\lambda+k}
$$


by the theorem of Späth for micro-differential operators. Here $Q_{i, k}\left(x^{\prime}\right)$ and $Q_{j, k}^{\prime}\left(x^{\prime}\right)$ are analytic functions with the variables $x_{3}, \ldots, x_{n}$ and $\lambda$ is a complex number. The equations

$$
\left[D_{x_{l}}, Q\left(x, D_{x}\right)\right] w(v)=0 \quad(l=3, \ldots, n)
$$

and

$$
\left[x_{1} D_{x_{1}}+\frac{1}{2} x_{2} D_{x_{2}}, Q\left(x, D_{x}\right)\right] w(v)=0
$$

entail that

$$
\begin{aligned}
0 & =\left[D_{x_{l}}, Q\left(x, D_{x}\right)\right] \\
& =\sum_{i, k} \frac{\partial Q_{i, k}}{\partial x_{l}}\left(x^{\prime}\right) s^{i} D_{x_{1}}^{\lambda+k}+\sum_{j, k} \frac{\partial Q_{j, k}^{\prime}}{\partial x_{l}}\left(x^{\prime}\right) t^{j} D_{x_{1}}^{\lambda+k}
\end{aligned}
$$

and that

$$
\begin{aligned}
0 & =\left[x_{1} D_{x_{1}}+\frac{1}{2} x_{2} D_{x_{2}}, Q\left(x, D_{x}\right)\right] \\
& =-\left(\sum_{i, k}(\lambda+k) Q_{i, k}\left(x^{\prime}\right) s^{i} D_{x_{1}}^{\lambda+k}+\sum_{j, k} Q_{j, k}^{\prime}\left(x^{\prime}\right) t^{j} D_{x_{1}}^{\lambda+k}\right),
\end{aligned}
$$

because $\left[D_{x_{l}}, Q\left(x, D_{x}\right)\right]$ and $\left[x_{1} D_{x_{1}}+\frac{1}{2} x_{2} D_{x_{2}}, Q\left(x, D_{x}\right)\right]$ are of the same form as $Q\left(x, D_{x}\right)$. Hence $Q\left(x, D_{x}\right) w(v)$ is of the form

$$
Q\left(x, D_{x}\right) w(v)=\left(\sum_{i=1}^{m} C_{i} s^{i}+\sum_{j=0}^{m^{\prime}} C_{j}^{\prime} t^{j}\right) w(v)
$$

with constants $C_{i}$ and $C_{j}^{\prime}$ such that either $C_{m}$ or $C_{m^{\prime}}^{\prime}$ is different from zero. If $-2 v \notin \mathbb{N}\left(2 v-1 \notin \mathbb{N}\right.$, resp.), we may replace $v$ by $v+\frac{m^{\prime}}{2}+2\left(v-\frac{m}{2}\right.$, resp. $)$ and $Q\left(x, D_{x}\right)$ by

$$
\begin{aligned}
& \sum_{i=1}^{m} C_{i} s^{m^{\prime}+2+i}+\sum_{j=0}^{m^{\prime}} C_{j}^{\prime} s^{m^{\prime}+2-j} \prod_{k=0}^{j-1}(-2 v-k) \\
& \left(\sum_{i=1}^{m} C_{i} t^{m-i} \prod_{k=0}^{i-1}(-2 v+1+k)+\sum_{j=0}^{m^{\prime}} C_{j}^{\prime} t^{m+j}, \text { resp. }\right),
\end{aligned}
$$

because $\mathfrak{N}_{-v-\frac{1}{2}, v-1}$ is isomorphic to

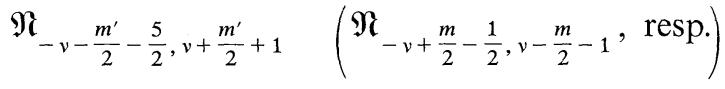

by the correspondence $w(v)=s^{m^{\prime}+2} w\left(v+\frac{m^{\prime}}{2}+2\right)\left(t^{m} w\left(v-\frac{m}{2}\right)\right.$, resp.) (cf. Lemma 2.2). Hence we may assume from the first that

$$
Q\left(x, D_{x}\right)=\sum_{i=m_{1}}^{m_{2}} C_{i} s^{i}
$$


or

$$
Q\left(x, D_{x}\right)=\sum_{j=m_{1}^{\prime}}^{m_{2}^{\prime}} C_{j}^{\prime} t^{j},
$$

where $m_{1} \geqq 2$ and $C_{m_{1}} \neq 0, C_{m_{2}} \neq 0, C_{m_{1}^{\prime}}^{\prime} \neq 0$ and $C_{m_{2}^{\prime}}^{\prime} \neq 0$.

Suppose (4.6). By the equation

$$
\begin{aligned}
((s- & \left.\left.\frac{1}{2} t\right) t s t-(\beta+\gamma) t^{2}+2(\alpha+\beta) s t+4 \alpha \beta\right) s^{i} w(v) \\
= & -\frac{1}{2}\left(s t^{3}-2\left(s^{2}-(\beta+\gamma+1)\right) t^{2}-2(2 \alpha+2 \beta+1) s t-8 \alpha \beta\right) s^{i} w(v) \\
= & -\frac{1}{2}\left(s^{i+1} t^{3}+\left(3 i s^{i}-2\left(s^{2}-(\beta+\gamma+1)\right) s^{i}\right) t^{2}+\left(3 i(i-1) s^{i-1}\right.\right. \\
& \left.-4\left(s^{2}-(\beta+\gamma+1)\right) i s^{i-1}-2(2 \alpha+2 \beta+1) s^{i+1}\right) t+\left(i(i-1)(i-2) s^{i-2}\right. \\
& \left.-2\left(s^{2}-(\beta+\gamma+1)\right) i(i-1) s^{i-2}-2(2 \alpha+2 \beta+1) i s^{i}-8 \alpha \beta s^{i}\right) w(v) \\
= & \left((i-2 v+2 \alpha)(i-2 v+2 \beta) s^{i}-\frac{1}{2}(-2 v(2 v+1)(2 v+2)+3 i(2 v)(2 v+1)\right. \\
& +2(\beta+\gamma+1)(2 v)(2 v+1)-3 i^{2}(2 v)-(4 \beta+4 \gamma+1) i(2 v)+i^{3}-3 i^{2}+2 i \\
& \left.\left.+2(\beta+\gamma+1) i^{2}-2(\beta+\gamma+1) i\right) s^{i-1}\right) w(v) \\
= & \left((i-2 v+2 \alpha)(i-2 v+2 \beta) s^{i}-\frac{1}{2}(i+2 \beta+2 \gamma-2 v)(i-2 v)(i-2 v-1) s^{i-2}\right) w(v)
\end{aligned}
$$

we have

$$
\begin{aligned}
0= & \sum_{i=m_{1}}^{m_{2}} C_{i}\left\{(i+2 \alpha-2 v)(i+2 \beta-2 v) s^{i}\right. \\
& \left.-\frac{1}{2}(i+2 \beta+2 \gamma-2 v)(i-2 v)(i-2 v-1) s^{i-2}\right\} w(v) .
\end{aligned}
$$

Hence we have

$$
\left\{\begin{array}{l}
C_{m_{2}}\left(m_{2}+2 \alpha-2 v\right)\left(m_{2}+2 \beta-2 v\right)=0, \\
C_{m_{1}}\left(m_{1}+2 \beta+2 \gamma-2 v\right)\left(m_{1}-2 v\right)\left(m_{1}-2 v-1\right)=0 .
\end{array}\right.
$$

Here we note that $m_{2}-m_{1}$ is a non-negative even integer. Therefore it follows from the assumptions $C_{m_{1}} \neq 0, C_{m_{2}} \neq 0, \gamma \notin \mathbb{N}$ and $\beta+\gamma-\alpha \notin \mathbb{N}$ that

$$
m_{2}+2 \beta-2 v=m_{1}-2 v=0 \quad \text { or } \quad m_{2}+2 \beta-2 v=m_{1}-2 v-1=0 .
$$

In this case, since $2 v$ is a positive integer and

$$
t^{2 v} s^{2 v} w(v)=s t(s t+1) \ldots(s t+2 v) w(v)=0,
$$

we have $s^{i} w(v)=0$ for $i \geqq 2 v$ on $\Lambda_{2}^{\mathbb{C}}-\Lambda_{1}^{\mathbb{C}}$ where $t$ is invertible. Hence $Q w(v)$ vanishes on $\Lambda_{2}^{\mathbb{C}}-\Lambda_{1}^{\mathbb{C}}$, which contradicts the fact that $h(\alpha, \beta, \gamma)=Q w(v)$ defines a surjective homomorphism of $\mathfrak{N}(\alpha, \beta, \gamma)$ to $\mathfrak{N}_{-v-\frac{1}{2}, v-1}$. 
On the other hand, suppose (4.7). Then by the same argument as in the case where (4.6) holds, we have

$$
\sum_{j=m_{1}^{\prime}}^{m_{2}^{\prime}} C_{j}^{\prime}\left\{\frac{1}{2} t^{2}(j-2 \beta-2 \gamma+2 v)+(j-2 \alpha+2 v)(j-2 \beta+2 v)\right\} t^{j} w(v)=0,
$$

and

$$
\left\{\begin{array}{l}
C_{m_{2}^{\prime}}^{\prime}\left(m_{2}^{\prime}-2 \beta-2 \gamma+2 v\right)=0, \\
C_{m_{1}^{\prime}}^{\prime}\left(m_{1}^{\prime}-2 \alpha+2 v\right)\left(m_{1}^{\prime}-2 \beta+2 v\right)=0 .
\end{array}\right.
$$

This contradicts the assumptions $C_{m_{1}^{\prime}}^{\prime} \neq 0, C_{m_{2}^{\prime}}^{\prime} \neq 0, \gamma \notin \mathbb{N}$ and $\beta+\gamma-\alpha \notin \mathbb{N}$ because $m_{2}^{\prime}-m_{1}^{\prime}$ is a non-negative even integer. Thus we have proved that Case 1 does not occur

Next study the possibility of Case 2 . Then the kernel $\mathfrak{N}^{\prime}$ of the natural homomorphism from $\mathfrak{M}(\alpha, \beta, \gamma)$ to $\mathfrak{N}$ is a simple holonomic system whose characteristic variety equals $\Lambda_{3}^{\mathbb{C}}$. Hence the same argument as in Case 1 proves that there exists a micro-differential operator $Q\left(x, D_{x}\right)$ of a fractional order such that the correspondence $w=Q\left(x, D_{x}\right) h(\alpha, \beta, \gamma)$ defines an injective homomorphism of the system

$$
\mathfrak{N}^{\prime \prime}:\left\{\begin{array}{l}
x_{1} w=0 \\
D_{x_{j}}=0, \quad j=2, \ldots, n
\end{array}\right.
$$

to the system $\mathfrak{P}(\alpha, \beta, \gamma)$ and moreover that we may assume $Q$ has the representation

$$
Q=\left(S_{1}\left(s^{\prime}\right)+T_{1}\left(t^{\prime}\right)\right)-\left(S_{2}\left(s^{\prime}\right)+T_{2}\left(t^{\prime}\right)\right) s^{\prime} t^{\prime},
$$

where $S_{i}$ and $T_{i}(i=1,2)$ are polynomials with one variable satisfying $T_{1}(0)$ $=T_{2}(0)=0$ and where $s^{\prime}=x_{2} D_{x_{1}}^{\frac{1}{2}}$ and $t^{\prime}=D_{x_{2}} D_{x_{1}}^{-\frac{1}{2}}$. Then the equation

$$
\begin{aligned}
s^{\prime}\{( & \left.\left.S_{1}\left(s^{\prime}\right)+T_{1}\left(t^{\prime}\right)\right)+\left(S_{2}\left(s^{\prime}\right)+T_{2}\left(t^{\prime}\right)\right) s^{\prime} t^{\prime}\right\} \\
= & S_{1}\left(s^{\prime}\right) s^{\prime}+\left(T_{1}\left(t^{\prime}\right) / t^{\prime}\right) s^{\prime} t^{\prime}+T_{1}\left(t^{\prime}\right) / t^{\prime}-d T_{1}\left(t^{\prime}\right) / d t^{\prime} \\
& +S_{2}\left(s^{\prime}\right) s^{\prime} s^{\prime} t^{\prime}+\left(T_{2}\left(t^{\prime}\right) / t^{\prime}\right) s^{\prime} t^{\prime} s^{\prime} t^{\prime}+\left(T_{2}\left(t^{\prime}\right) / t^{\prime}\right) s^{\prime} t^{\prime} \\
& \quad-\left(d T_{2}\left(t^{\prime}\right) / d t^{\prime}\right) s^{\prime} t^{\prime}
\end{aligned}
$$

and the equation

$$
s^{\prime} t^{\prime} s^{\prime} t^{\prime} h(\alpha, \beta, \gamma)=\left(-\frac{1}{2} t^{\prime 2} s^{\prime} t^{\prime}+(\gamma-\alpha+1) t^{\prime 2}-2(\alpha+\beta) s^{\prime} t^{\prime}-4 \alpha \beta\right) h(\alpha, \beta, \gamma)
$$

entail that

$$
\left\{\begin{array}{l}
s^{\prime} S_{1}\left(s^{\prime}\right)=0 \\
\left(T_{1}\left(t^{\prime}\right) / t^{\prime}\right)-d T_{1}\left(t^{\prime}\right) / d t^{\prime}+(\gamma-\alpha+1) t^{\prime} T_{2}\left(t^{\prime}\right)-4 \alpha \beta T_{2}\left(t^{\prime}\right) / t^{\prime}=0 \\
s^{\prime} S_{2}\left(s^{\prime}\right)=0 \\
\left(T_{1}\left(t^{\prime}\right) / t^{\prime}\right)-\frac{1}{2} t^{\prime} T_{2}\left(t^{\prime}\right)-2(\alpha+\beta) T_{2}\left(t^{\prime}\right) / t^{\prime}+T_{2}\left(t^{\prime}\right) / t^{\prime}-d T_{2}\left(t^{\prime}\right) / d t^{\prime}=0
\end{array}\right.
$$


because $s^{\prime} Q h(\alpha, \beta, \gamma)=0$. Hence $S_{1}=S_{2}=0$ and

$$
\left\{\begin{array}{l}
\left(t^{\prime} d / d t^{\prime}-1\right) T_{1}\left(t^{\prime}\right)+\left(4 \alpha \beta-(\gamma-\alpha+1) t^{\prime 2}\right) T_{2}\left(t^{\prime}\right)=0 \\
T_{1}\left(t^{\prime}\right)-\left(t^{\prime} d / d t^{\prime}+\left\{(2 \alpha+2 \beta-1)+\frac{1}{2} t^{\prime 2}\right\}\right) T_{2}\left(t^{\prime}\right)=0 .
\end{array}\right.
$$

Therefore we have

$$
\frac{1}{2}\left(t^{\prime} d / d t^{\prime}+2 \alpha-2 \gamma-3\right) t^{\prime 2} T_{2}\left(t^{\prime}\right)+\left(t^{\prime} d / d t^{\prime}+2 \alpha-1\right)\left(t^{\prime} d / d t^{\prime}+2 \beta-1\right) T_{2}\left(t^{\prime}\right)=0 .
$$

Since $T_{2}$ has a representation

$$
T_{2}\left(t^{\prime}\right)=\sum_{i=0}^{m^{\prime}} C_{i} t^{\prime m+2 i}
$$

with constant numbers $C_{i}$ satisfying $C_{0} \neq 0$ and $C_{m^{\prime}} \neq 0$, we have

$$
\left\{\begin{array}{l}
m+2 m^{\prime}+2+2 \alpha-2 \gamma-3=0 \\
(m+2 \alpha-1)(m+2 \beta-1)=0
\end{array}\right.
$$

Therefore

$$
1-2 \alpha \in \mathbb{N}, \quad \gamma \in \mathbb{N}
$$

or

$$
1-2 \beta \in \mathbb{N}, \quad \beta+\gamma-\alpha \in \mathbb{N} .
$$

This contradicts the assumption.

Thus we have proved Lemma 4.7 on condition that our conjecture is true.

C-Remark 4.10. We consider the case when Condition (4.5) does not hold in Theorem 4.1. In $\S 1$ we proved that the hyperfunction $f$ is a sum of an analytic function and multiplications of hyperfunctions of the forms (1.14)-(1.16) and (1.17) by analytic functions. If $f$ is represented as a sum of an analytic function and multiplications of hyperfunctions of the forms (1.14) and (1.15), then Theorem 4.6 holds without Condition (4.5) because the same proof as that of Theorem 4.6 with Condition (4.5) is valid.

C-Corollary. The characteristic variety of any holonomic system of microdifferential equations that the Feynman amplitude $f_{D_{1}}(p)$ considered in twodimensional world contains $\Lambda_{3}^{\mathbb{C}}$ as its components. The same statement holds for $f_{D_{2}}(p)$ considered in four-dimensional world.

This corollary immediately follows from Remark 4.10 for $f_{D_{1}}(p)$ or from Theorem 4.6 for $f_{D_{2}}(p)$. Note that the formula of the order $\alpha(D)$ of the holonomic system in question (Kawai and Stapp [10]) must be changed to

$$
\frac{v}{2} n^{\prime}+\left(\frac{1}{2}-\frac{v}{2}\right) N
$$

in the $v$-dimensional world. 
Acknowledgement. It is a great pleasure of the authors to recall with heartiest thanks the illuminating and encouraging discussions which the authors enjoyed with Professor M. Sato, Professor H. P. Stapp, and Professor T. Regge on the topics dealt with in this paper.

\section{References}

1. Branson, D.: Nuovo Cimento 54 A, 217-233 (1968)

2. Chandler,C., Stapp,H.P.: J. Math. Phys. 10, 826-859 (1969)

3. Eden, R.J.: Phys. Rev. 121, 1567-1576 (1961)

4. Eden,R.J., Landshoff,P.V., Olive,D.I., Polkinghorne,J.C.: The analytic $S$-matrix. Cambridge: Cambridge University Press 1966

5. Kashiwara, M., Kawai,T.: J. Math. Soc. Japan 27, 359-404 (1975)

6. Kashiwara,M., Kawai,T.: Publ. RIMS Kyoto Univ. 12, Suppl., 131-140 (1977)

7. Kashiwara, M., Kawai,T.: Holonomic character and local monodromy structure of Feynman integrals. Commun. math. Phys. 54, 121-134 (1977)

8. Kashiwara,M., Kawai,T.: Holonomic character and local monodromy structure of Feynman integrals. II. (in preparation)

9. Kashiwara,M., Kawai,T., Stapp,H.P.: Publ. RIMS Kyoto Univ. 12, Suppl., 141-146 (1977)

10. Kawai, T., Stapp, H.P.: Publ. RIMS Kyoto Univ. 12, Suppl., 155-232 (1977)

11. Landshoff,P.V., Olive, D.I., Polkinghorne,J.C.: Nuovo Cimento 43 A, 444-453 (1966)

12. Landshoff,P.V., Polkinghorne,J.C., Taylor,J.C.: Nuovo Cimento 19, 939-952 (1961)

13. Nakanishi,N.: Graph theory and Feynman integrals. New York-London-Paris: Gordon and Breach 1971

14. Oshima, T.: J. Fac. Sci. Univ. Tokyo Sec. IA 21, 43-83 (1974)

15. Pham, F.: Ann. Inst. H. Poincaré 6 A, 89-204 (1967)

16. Regge, T.: Algebraic topology methods in the theory of Feynman relativistic amplitudes. In: Report of battle recountres, pp. 433-458. New York: Benjamin 1968

17. Regge,T.: Feynman integrals. In: Actes Congrès Intern. Math. (Nice), Tome 3, pp.9-14. Paris: Gauthier-Villars 1970

18. Regge,T., Speer, E. R., Westwater,M.J.: Fortschr. Phys. 20, 365-420 (1972)

19. Sato, M.: Recent development in hyperfunction theory and its application to physics. Lecture notes in physics, Vol. 39, pp. 13-29. Berlin-Heidelberg-New York: Springer 1975

20. Sato,M., Kawai,T., Kashiwara,M.: Microfunctions and pseudo-differential equations. Lecture notes in mathematics, Vol. 287, pp. 265-529. Berlin-Heidelberg-New York: Springer 1973

21. Speer,E.R.: Generalized Feynman amplitudes. Princeton: Princeton University Press 1969

22. Speer,E. R., Westwater,M.F.: Ann. Inst. H. Poincaré 14 A, 1-55 (1971)

23. Westwater, M.J.: Helv. Phys. Acta 40, 389-400 (1967)

Communicated by H. Araki

Received August 30, 1977; in revised form November 2, 1977 